Revised Manuscript \# MSSP 16-508R1 Submitted in November 2016

\title{
VIBRATION CONTROL OF A CYLINDRICAL SHELL WITH CONCURRENT ACTIVE PIEZOELECTRIC PATCHES AND PASSIVE CARDBOARD LINER
}

\author{
Joseph Plattenburg, Jason T. Dreyer, Rajendra Singh* \\ Acoustics and Dynamics Laboratory, NSF Smart Vehicles Concepts Center \\ Department of Mechanical and Aerospace Engineering, \\ The Ohio State University, Columbus, OH 43210, USA \\ *Corresponding Author: E-mail: singh.3@osu.edu; Phone: 614-292-9044; Fax: 614-292-3163
}

\begin{abstract}
This article extends a recent publication [MSSP (2016), 176-196] by developing a Rayleigh-Ritz model of a thin cylindrical shell to predict its response subject to concurrent active and passive damping treatments. These take the form of piezoelectric patches and a distributed cardboard liner, since the effects of such combined treatments are yet to be investigated. Furthermore, prior literature typically considers only the "bimorph" active patch configuration (with patches on the inner and outer shell surfaces), which is not feasible with an interior passive liner treatment. Therefore, a novel configuration-termed as "unimorph"-is proposed and included in the model. Experiments are performed on a shell with active patches (under harmonic excitation from 200 to $2000 \mathrm{~Hz}$ ) in both the bimorph and unimorph configurations to provide evidence for the analytical model predictions. The proposed model is then employed to assess competing control system designs by examining local vs. global control schemes as well as considering several alternate active patch locations, both with and without the passive damping. Non-dimensional performance metrics are devised to facilitate comparisons of vibration attenuation among different designs. Finally, insertion loss values are measured under single-frequency excitation to evaluate several vibration control designs, and to compare the effects of alternate damping treatments.
\end{abstract}

Keywords: Shell Dynamics, Modal Analysis, Piezoelectric Patches, Distributed Damping Liners, Hybrid Damping, Vibro-acoustic Control 


\section{Introduction}

In a recent article [1], the authors developed a semi-analytical model to predict the vibration of a thin cylindrical shell subject to a homogenous cardboard damping liner. The motivation for the prior paper was the lack of physics-based analytical studies, despite the common usage of such damping liners for noise reduction in automotive drive shafts [2-5]. Aside from these empirical or computational studies, few other publications exist for lined shell systems outside of the recent article [1] and an experimental study by Koruk et al. [6]. The chief goals of this companion article are to extend the prior article [1] by proposing a semi-analytical model for a thin cylindrical shell with concurrent active (piezoelectric patches) and passive (distributed cardboard liner) damping treatments. Given a practical need for exploring lightweight vibro-acoustic treatments for vehicle applications [2-6], this article aims to develop a mathematical framework for the evaluation of vibration control strategies.

Active patches have been used extensively for vibration control of shells, e.g. by Sonti and Jones [7, 8] and Chaudhry et al. [9], who proposed the analytical models to predict shell response due to patch input. These investigators typically assume an active patch on both the inside and outside shell surface, termed as the "bimorph" configuration. Other experimental work using active patches or distributed actuators includes publications by Fuller et al. [10], Han and Lee [11], and Bailey and Hubbard [12], for instance. There is also a significant body of literature on active vibration control systems, as evident from literature review by Cabell and Fuller [13] and Kwak et al. [14]. However, relatively fewer publications have addressed combined active and passive control methods for thin shells [15], though prior methods have considered the active constrained layer damping approach in-depth [16-18]. Nevertheless, combined (yet independent) active and passive damping treatments have attracted only limited attention. For example, studies were published on a beam and a thin plate by Lam et al. [19] and Plattenburg et al. [20], respectively, but no similar studies seem to exist for cylindrical shells in terms of concurrent active and passive damping. Based on the above mentioned voids in the literature, this article intends to develop a refined model (as an extension of prior work [1]), for the novel "unimorph" configuration, using a single 
active patch, that would modify the existing bimorph theory [7, 9]. Experimental evidence of the model refinements will be included, and a few vibration control strategies will be discussed.

\section{Problem formulation}

Consider a thin cylindrical shell of length $l$, thickness $h$, and radius $a$ (from the central axis to the mid-plane of the shell) as depicted in Figure 1, with the assumed cylindrical coordinate system as shown. The shell is of elastic material with Young's modulus $E$, mass density $\rho$, Poisson's ratio $v$, and structural loss factor $\eta$ (see Appendix A for the identification of symbols). The system may also have $N_{c}$ piezoelectric active patches as shown in Figure 1, used to exert control input $\varepsilon_{c}$. The $r$ th patch is located at $\left(x_{c}^{r}, \theta_{c}{ }^{r}\right)$ relative to the coordinate system, and has thickness $h_{c}{ }^{r}$ and size $l_{x c}{ }^{r} \times l_{\theta c}{ }^{r}$. As from [1], the system may also have an internal cardboard liner of thickness $h_{L}$ with length $l_{L}=l$ and radius $a_{L}=a-h_{m}$ (interference fit with "preload") where $h_{m}=\left(h+h_{L}\right) / 2$. The liner, like the shell material, has properties $E_{L}$, $\rho_{L}, v_{L}$, and $\eta_{L}$ (where $E_{L}$ and $\eta_{L}$ may be frequency dependent). The shell liner interface also has an associated viscous friction coefficient, $c(\omega)$, possibly having frequency dependence. While Plattenburg et al. [1] investigated both viscous and Coulomb interfacial formulations, viscous damping was found to be the dominant mechanism and thus it will be the only interfacial interaction retained for this study. Finally, a disturbance force, $F_{d}$ may be applied to the shell (assumed normal to the surface) at $\left(x_{d}, \theta_{d}\right)$ and a measurement of out-of-plane motion, $w$, made at location $\left(x_{0}, \theta_{0}\right)$. Free boundaries (as shown in Figure 1) are assumed for the scope of this study as they may be accurately replicated experimentally. Material properties and dimensions for this system are given in Table 1.

Prior work for shell resonance control includes many studies on passive constrained layer damping using analytical and computational methods [21-23], cardboard liners with experimental methods for vehicle applications [2-6], and active piezoelectric patches with analytical, computational, and experimental methods [7-9, 14]. In particular, the piezoelectric patches are typically used in the "bimorph" configuration [7], with a patch on the inner and outer shell surface where they can be actuated in-phase (Figure 2a) or out-of-phase (Figure 2b). The two phasing configurations can be approximately 
modeled by an in-plane traction-like loading or an out-of-plane moment-like loading, respectively [7, 9], but some complicating coupling effects (including an effective distributed pressure) may arise due to the shell curvature. If a cardboard liner were to be used for passive damping, the bimorph configuration would not be possible and a "unimorph" configuration would instead need to be employed for combined active-passive damping (as shown in Figure 2c). To the authors' knowledge, this particular configuration has never been studied, especially when combined with a liner. A modified theoretical approximation will be presented for this case based on the work of the prior researchers [7, 9].

The objectives of this paper are as follows. First, develop a new semi-analytical model for vibration of shell structures subject to concurrent active and passive damping treatments, consisting of a distributed damping liner and piezoelectric active patches (using the unimorph configuration). Such a model would extend the work of prior researchers on the dynamic analysis of shells and active/passive damping [1, 6-9]. Second, conduct experimental studies to validate the predictions of this model (for both unimorph and bimorph configurations). Third, employ the model to assess competing vibration control system strategies subject to design constraints, examining local and global control schemes, and exploring several active patch locations and configurations, both with and without the damping liner. The control measures will be evaluated in terms of insertion loss at specific shell modes as well as using new performance metrics in the frequency range 200 to $2000 \mathrm{~Hz}$, where distinct shell resonances are observed in practical applications [2-6]. Experimental studies of insertion losses at a specific shell mode (single frequency) will also be provided for selected designs.

Like the prior article, the proposed analytical technique is the Rayleigh-Ritz method for prediction of shell vibration with free boundaries assumed at $x=0$ and $x=l$ for $0 \leq \theta<2 \pi$. Active patches are macro-fiber composite (MFC) piezoceramic actuators $[14,24]$ and assumed to be bonded perfectly to the shell surface (implying continuity of strain). A thin cardboard liner will be used with no adhesion, but rather an assumed interference fit such that the liner and shell remain in contact under out-of-plane motion with $w>0$ [1]. Energy methods and Lagrange's equation are used to derive the governing equations, and analysis is completed assuming steady state harmonic response in the frequency-domain 
(at $\omega,[\mathrm{rad} / \mathrm{s}]$ ). Frequency-dependent properties such as $E_{L}(\omega)$ and $\eta_{L}(\omega)$ may be used for the cardboard liner, but their temperature dependence is outside the scope of this research. Other assumptions include the following. Active patches have negligible bonding layer thickness and do not appreciably affect the inertial and elastic distribution of the substructure $(\rho(x, \theta)$ and $E(x, \theta)$, respectively). A simplified form of the equivalent patch loading is used which requires the assumption of equality of Poisson's ratios, $v \approx$ $v_{c}$, which appears reasonable from Table 1. Shear deformations and rotary inertia of structural elements (shell and damping liner) are taken to be negligible. Finally, the shell-liner system is assumed to be governed by Flügge's thin shell theory [25].

\section{Development of semi-analytical model}

The primary changes from the prior Rayleigh-Ritz model (of [1]) are inclusion of forcing from the active patches (detailed in Section 4.1) and a generalization of circumferential shape functions to allow for arbitrarily located patches. Therefore, two orthogonal sets of circumferential shape functions [26], namely $\sin (n \theta)$ and $\cos (n \theta)$, are defined as:

$$
\begin{aligned}
\boldsymbol{\Phi}_{c}^{w}(x, \theta)=\left\{\begin{array}{llll}
\phi_{c, 1}^{w}(x, \theta) & \phi_{c, 2}^{w}(x, \theta) & \ldots & \phi_{c, N_{s}}^{w}(x, \theta)
\end{array}\right\}^{\mathrm{T}} \\
\phi_{c, k}^{w}(x, \theta)=X_{m}(x) \cos (n \theta) \\
\boldsymbol{\Phi}_{s}^{w}(x, \theta)=\left\{\begin{array}{llll}
\phi_{s, 1}^{w}(x, \theta) & \phi_{s, 2}^{w}(x, \theta) & \ldots & \phi_{s, N_{s}}^{w}(x, \theta)
\end{array}\right\}^{\mathrm{T}} \\
\\
\phi_{s, k}^{w}(x, \theta)=X_{m}(x) \sin (n \theta) .
\end{aligned}
$$

Here, $\phi$ are 2-dimensional shape functions (grouped into vectors $\boldsymbol{\Phi}), X_{m}$ are axial (beam-like) shape functions (adapted from $[1,27]$ ), superscript $w$ refers to transverse motion, and subscripts $s$ and $c$ refer to sine and cosine circumferential shape functions, respectively. The $N_{s}$ shape functions result in an assumed form of the transverse motion, $w$ (see Figure 1), via a Ritz linear combination as:

$$
w(x, \theta)=\left[\mathbf{q}_{s}^{w}\right]^{\mathrm{T}} \mathbf{\Phi}_{s}^{w}(x, \theta)+\left[\mathbf{q}_{c}^{w}\right]^{\mathrm{T}} \mathbf{\Phi}_{c}^{w}(x, \theta),
$$

where temporal dependence (assumed harmonic, with angular frequency $\omega[\mathrm{rad} / \mathrm{s}]$ ) is dropped for clarity. The formulations for other motion types (e.g. in-plane, $u, v$; and liner motions, $u_{L}$ and $v_{L}$ ) have analogous 
definitions. The weighting coefficients and all the $\boldsymbol{\Phi}^{\alpha}$ are assembled into vectors of size $\kappa N_{s} \times 1$ (where $\kappa$ $=5$ is the dimension of the system):

$$
\boldsymbol{\Phi}=\left\{\begin{array}{lllll}
\boldsymbol{\Phi}^{w \mathrm{~T}} & \boldsymbol{\Phi}^{u \mathrm{~T}} & \boldsymbol{\Phi}^{v \mathrm{~T}} & \boldsymbol{\Phi}^{u_{L} \mathrm{~T}} & \boldsymbol{\Phi}^{v_{L} \mathrm{~T}}
\end{array}\right\}^{\mathrm{T}}, \quad \mathbf{q}=\left\{\begin{array}{lllll}
\mathbf{q}^{w \mathrm{~T}} & \mathbf{q}^{u \mathrm{~T}} & \mathbf{q}^{v \mathrm{~T}} & \mathbf{q}^{u_{L} \mathrm{~T}} & \mathbf{q}^{v_{L} \mathrm{~T}}
\end{array}\right\}^{\mathrm{T}} .
$$

Note that if no liner is used (i.e. the reduced case from [1]) then $\kappa=3$. Applying Lagrange's equation to the energies and non-conservative work with respect to the unknown weighting factors, $\mathbf{q}$, results in the following system of equations:

$$
\mathbf{M} \ddot{\mathbf{q}}+\mathbf{C} \dot{\mathbf{q}}+\tilde{\mathbf{K}} \mathbf{q}=\mathbf{0},
$$

Here $\tilde{\mathbf{K}}\left(\in \square^{\kappa N_{s} \times \kappa N_{s}}\right), \mathbf{C}$, and $\mathbf{M}\left(\in \square^{\kappa N_{s} \times \kappa N_{s}}\right)$ are equivalent stiffness, damping, and mass matrices given by Plattenburg et al. [1]. Because sine and cosine integrate to the same value over full periods, only one of the two orthogonal sets need be retained in Eq. (3a) and in the integrals generating $\mathbf{M}, \mathbf{C}$, and $\tilde{\mathbf{K}}$; however these will play a more important role in Section 4 and beyond with external forcing considered.

The complex eigenvalue problem associated with Eq. (4) can be solved by standard methods [1], and the natural frequencies and modal loss factors of a sample system (having dimensions and material properties given in Table 1 and damping coefficient $c_{v}=1 \times 10^{5} \mathrm{~kg}-\mathrm{m}^{-2} \mathrm{~s}^{-1}$ ) are denoted for selected modes in Table 2. Note that the presence of the liner typically results in a decrease in natural frequency $\left(f_{i}\right)$ as well as an increase in modal loss factor $\left(\eta_{i}\right)$ and peak attenuation (insertion loss).

\section{Forced harmonic responses from active patches and disturbance}

\subsection{Effective loading from active patches}

In order to account for the inputs to the shell system from the active patches, the Rayleigh-Ritz method requires a generalized forcing term [28] to be added to the right-hand side of Eq. (4) as:

$$
\mathbf{Q}^{\alpha}(t)=a_{i} \int_{0}^{L_{x}} \int_{0}^{2 \pi} F^{\alpha}(x, \theta, t) \mathbf{\Phi}^{\alpha}(x, \theta) d \theta d x
$$

where $\alpha$ refers to one of the $\kappa$ sets of shape functions and $F^{\alpha}$ refers to a distributed force component corresponding to one of those motions (i.e. a force normal to the surface corresponds to $w$, traction in the 
axial direction corresponds to $u$, etc.). Following Sonti and Jones [7] and Chaudhry et al. [9] for bimorph active patch configurations, the out-of-phase case is represented by line moments along the patch boundaries and the in-phase case by line forces at the patch boundaries plus a distributed pressure over the patch area. These equivalent loading vectors can be written for the $r$ th active patch (assuming harmonic excitation at frequency $\omega$ ) for the out-of-phase case as:

$$
\begin{array}{r}
\tilde{\vec{F}}_{c, \text { out }}^{r}(x, \theta, t)=\left\{M_{x}^{r}\left[\delta^{\prime}\left(x-x_{c 1}^{r}\right)-\delta^{\prime}\left(x-x_{c 2}^{r}\right)\right]\left[H\left(\theta-\theta_{c 1}^{r}\right)-H\left(\theta-\theta_{c 2}^{r}\right)\right]+\ldots\right. \\
\left.M_{\theta}^{r}\left[H\left(x-x_{c 1}^{r}\right)-H\left(x-x_{c 2}^{r}\right)\right]\left[\delta^{\prime}\left(\theta-\theta_{c 1}^{r}\right)-\delta^{\prime}\left(\theta-\theta_{c 2}^{r}\right)\right]\right\} \hat{e}_{r} \mathrm{e}^{\mathrm{j}\left(\omega t+\varphi_{c}^{r}\right)},
\end{array}
$$

and for the in-phase case as:

$$
\begin{gathered}
\tilde{\vec{F}}_{c, i n}^{r}(x, \theta, t)=\left(\tilde{F}_{x}^{r}(x, \theta) \hat{e}_{x}+\tilde{F}_{\theta}^{r}(x, \theta) \hat{e}_{\theta}+\tilde{F}_{r}^{r}(x, \theta) \hat{e}_{r}\right) \mathrm{e}^{\mathrm{j}\left(\omega t+\varphi_{c}^{r}\right)}, \\
\tilde{F}_{x}^{r}(x, \theta)=\tilde{F}_{x}^{r}\left[\delta\left(x-x_{c 2}^{r}\right)-\delta\left(x-x_{c 1}^{r}\right)\right]\left[H\left(\theta-\theta_{c 1}^{r}\right)-H\left(\theta-\theta_{c 2}^{r}\right)\right], \\
\tilde{F}_{\theta}^{r}(x, \theta)=\tilde{F}_{\theta}^{r}\left[\delta\left(\theta-\theta_{c 2}^{r}\right)-\delta\left(\theta-\theta_{c 1}\right)\right]\left[H\left(x-x_{c 1}^{r}\right)-H\left(x-x_{c 2}^{r}\right)\right], \\
\tilde{F}_{r}^{r}(x, \theta)=\tilde{P}_{r}^{r}\left[H\left(x-x_{c 1}^{r}\right)-H\left(x-x_{c 2}^{r}\right)\right]\left[H\left(\theta-\theta_{c 1}^{r}\right)-H\left(\theta-\theta_{c 2}^{r}\right)\right] .
\end{gathered}
$$

Here, $F$ is a 3 -dimensional force vector with components in the $x, \theta$, and $r$ directions; $\hat{e}_{x}, \hat{e}_{\theta}$, and $\hat{e}_{r}$ are unit vectors spanning $\square^{3}$ in cylindrical coordinates; $\delta(\cdot)$ is the Dirac delta function; $H(\cdot)$ is the Heaviside function; $\varphi_{c}$ is the control input phase relative to a reference; $x_{c 1}, x_{c 2}, \theta_{c 1}$, and $\theta_{c 2}$ are patch boundaries (i.e. $x_{c} \pm 0.5 L_{x, c}$ and $\left.\theta_{c} \pm 0.5 L_{\theta, c} / a\right) ; M_{x}$ and $M_{\theta}$ are equivalent moment strengths from the active patches; $F_{x}$ and $F_{\theta}$ are equivalent traction line forces; and $P_{r}$ is the equivalent distributed pressure. This pressure is given as $P_{r}=-F_{\theta} / a$, obtained from static force balance on the shell sector beneath the active patch. This equivalent pressure (illustrated in Figure 3) is given in the literature [7,9] in order to avoid a rigid body loading resulting from the equivalent traction forces with a nonzero resultant.

Note that the approximate moment induced by an active patch pair in the out-of-phase case is transformed to an equivalent force-couple in Eq. (7) as from [29] (via the derivative of the Dirac delta function, $\delta^{\prime}(\cdot)$ ). Schematics of the equivalent loadings from Eqs. (6) and (7) are shown in Figures 4a and 4b. Next, it is proposed that the net loading of the unimorph active patch configuration could be 
approximated as a combination of the in-phase and out-of-phase bimorph configurations. The single patch induces in-plane traction and pressure on the surface, and since it is offset from the mid-plane of the shell there is also a net effective moment at the patch boundaries. This can be seen intuitively from Figure 1, where the equivalent loading in (c) is equal to the resultant of half the sum of (a) and (b).

Here a simplified piezoelectric model [7,9] is used with an assumed relation between piezoelectric strain and voltage of the form $\varepsilon_{c}=d_{31} V_{c}$, where $d_{31}$ is a generic (potentially non-constant) piezoelectric strain-voltage coefficient. For example, Crawley and Anderson [30] detail some of the nonlinearities that may exist with these coefficients. Under these assumptions, $F_{x}$ and $F_{\theta}$ are given as:

$$
\tilde{F}_{x}=\left(\frac{E h}{1-v}\right) \cdot\left(\frac{2 E_{c} h_{c}}{2 E_{c} h_{c}+E h}\right) \cdot \tilde{V}_{c}\left(d_{31}-v_{c} d_{32}\right), \quad \tilde{F}_{\theta}=\left(\frac{E h}{1-v}\right) \cdot\left(\frac{2 E_{c} h_{c}}{2 E_{c} h_{c}+E h}\right) \cdot \tilde{V}_{c}\left(d_{32}-v_{c} d_{31}\right),
$$

each having units of [N/m]. Moment loading magnitudes, $M_{x}$ and $M_{\theta}$ are written as:

$$
\begin{aligned}
& \tilde{M}_{x}=\left(\frac{E h^{2}}{1-v}\right) \cdot\left(\frac{E_{c} h_{c} h\left(h+h_{c}\right)}{E h^{3}+E_{c} h_{c}\left(6 h^{2}+12 h_{c} h+8 h_{c}^{2}\right)}\right) \cdot \tilde{V}_{c}\left(d_{31}-v_{c} d_{32}\right), \\
& \tilde{M}_{\theta}=\left(\frac{E h^{2}}{1-v}\right) \cdot\left(\frac{E_{c} h_{c} h\left(h+h_{c}\right)}{E h^{3}+E_{c} h_{c}\left(6 h^{2}+12 h_{c} h+8 h_{c}^{2}\right)}\right) \cdot \tilde{V}_{c}\left(d_{32}-v_{c} d_{31}\right),
\end{aligned}
$$

and the distributed pressure, $P_{r}$, is obtained from $F_{\theta}$ as above. Here, $\tilde{V}_{c}$ is the complex-valued control voltage, $d_{31}$ and $d_{32}$ piezoelectric coefficients aligned in the $x$ and $\theta$ directions, respectively, and the negative Poisson term $(v)$ is due to the effective compression induced by the Poisson effect. Typically, MFC patches actuate uni-axially, so $d_{32}$ will be assumed to be zero. A schematic of the resulting configuration and equivalent loading is shown in Figure 5c.

Thus all the loading coefficients are obtained in terms of the excitation and material properties, and substituting Eqs. (8) and (9) for unimorph loading into Eqs. (6) and (7) and the result into Eq. (5) gives the following expression for $\mathbf{Q}_{c}$ : 


$$
\begin{aligned}
& \tilde{\mathbf{Q}}_{c}=\left\{\begin{array}{lllll}
\tilde{\mathbf{Q}}_{c}^{w \mathrm{~T}} & \tilde{\mathbf{Q}}_{c}^{u \mathrm{~T}} & \tilde{\mathbf{Q}}_{c}^{v \mathrm{~T}} & \mathbf{0} & \mathbf{0}
\end{array}\right\}^{\mathrm{T}}, \\
& \tilde{Q}_{c, k}^{w}=\left\{a \tilde{M}_{x}\left[X_{m}^{\prime}\left(x_{1}\right)-X_{m}^{\prime}\left(x_{2}\right)\right]\left[\int_{\theta_{1}}^{\theta_{2}} \Theta_{n}(\theta) d \theta\right]+\ldots\right. \\
& \left.\frac{1}{a} \tilde{M}_{\theta}\left[\int_{x_{1}}^{x_{2}} X_{m}(x) d x\right]\left[\Theta_{n}^{\prime}\left(\theta_{1}\right)-\Theta_{n}^{\prime}\left(\theta_{2}\right)\right]+a \tilde{P}_{r}\left[\int_{x_{2}}^{x_{2}} X_{m}(x) d x\right]\left[\int_{\theta_{1}}^{\theta_{2}} \Theta_{n}(\theta) d \theta\right]\right\} \mathrm{e}^{\mathrm{j} \varphi_{c}}, \\
& \tilde{Q}_{c, k}^{u}=a \tilde{F}_{x}\left[X_{m}\left(x_{2}\right)-X_{m}\left(x_{1}\right)\right]\left[\int_{\theta_{1}}^{\theta_{2}} \Theta_{n}(\theta) d \theta\right] \mathrm{e}^{\mathrm{j} \varphi_{c}}, \\
& \tilde{Q}_{c, k}^{v}=\tilde{F}_{\theta}\left[\int_{x_{2}}^{x_{2}} X_{m}(x) d x\right]\left[\Theta_{n}\left(\theta_{2}\right)-\Theta_{n}\left(\theta_{1}\right)\right] \mathrm{e}^{\mathrm{j} \varphi_{c}} .
\end{aligned}
$$

Here, $X_{m}(x)$ and $\Theta_{n}(\theta)$ are the $m$ th and $n$th axial and circumferential shape functions in the notation of [1] (of a total of $N_{x}$ and $N_{\theta}$ shape functions, with $N_{x} \times N_{\theta}=N_{s}$ ) and $k$ refers to the $k$ th element of the $\tilde{\mathbf{Q}}$ vector, with mapping $k=(m-1) N_{\theta}+n$. This may be readily extended to the case of $N_{c}$ active patches, each with different relative phasing, $\varphi_{c}{ }^{r}$ and voltage input, $V_{c}{ }^{r}$. in which case each of the $\tilde{\mathbf{Q}}$ component vectors are summed over $r=1, \ldots, N_{c}$. This can be conveniently written in the form $\tilde{\mathbf{Q}}_{c}=\mathbf{B}_{c} \tilde{\mathbf{u}}_{c}$, where $\tilde{\mathbf{u}}_{c}=\left\{\begin{array}{lll}\tilde{V}_{c}^{1} & \ldots & \tilde{V}_{c}^{N_{c}}\end{array}\right\}^{\mathrm{T}}$ is a vector of complex-valued control inputs and $\mathbf{B}_{c}$ is an input matrix mapping containing information about each individual patch's geometry, location, and material properties (without the magnitude and phase dependence associated with the input voltage). The matrix $\mathbf{B}_{c}\left(\in \square^{\kappa N_{s} \times N_{c}}\right)$ is defined as follows:

$$
\begin{aligned}
& \mathbf{B}_{c}=\left[\begin{array}{lllll}
\mathbf{B}_{c}^{w \mathrm{~T}} & \mathbf{B}_{c}^{u \mathrm{~T}} & \mathbf{B}_{c}^{v \mathrm{~T}} & \mathbf{0} & \mathbf{0}
\end{array}\right]^{\mathrm{T}}, \\
& \mathbf{B}_{c}^{w}=\left\{\begin{array}{lll}
\mathbf{B}_{c}^{w, 1} & \cdots & \mathbf{B}_{c}^{w, N_{c}}
\end{array}\right\}, \\
& \mathbf{B}_{c}^{w, r}=\frac{\tilde{\mathbf{Q}}_{c}^{w, r}}{V_{c} \mathrm{e}^{\mathrm{j} \varphi_{c}^{r}}}=\frac{\tilde{\mathbf{Q}}_{c}^{w, r}}{\tilde{V}_{c}^{r}}, \quad \mathbf{B}_{c}^{u, r}=\frac{\tilde{\mathbf{Q}}_{c}^{u, r}}{\tilde{V}_{c}^{r}}, \quad \mathbf{B}_{c}^{v, r}=\frac{\tilde{\mathbf{Q}}_{c}^{v, r}}{\tilde{V}_{c}^{r}} .
\end{aligned}
$$

Such a representation will be useful for control scheme derivations in Section 5. It should also be noted that because the harmonic inputs (disturbance or control) are typically localized, the limits on the $\theta$ integral in Eq. (5) effectively become different than 0 and $2 \pi$, integrals of $\Theta(\theta)$ are no longer over full 
periods of sine and cosine, and thus Eq. (5) assumes a different value depending on whether $\boldsymbol{\Phi}_{s}(x, \theta)$ or $\boldsymbol{\Phi}_{c}(x, \theta)$ is used. Ultimately, two forcing vectors, $\tilde{\mathbf{Q}}_{c}$ and $\tilde{\mathbf{Q}}_{c, o}$ (subscript $o$ indicating orthogonal) must be generated (corresponding to substitution of $\boldsymbol{\Phi}_{s}$ and $\boldsymbol{\Phi}_{c}$ of Eq. (1) into Eq. (5), respectively), and therefore, two input matrices, $\mathbf{B}_{c}$ and $\mathbf{B}_{c, o}$, are also generated.

\subsection{Effective loading from disturbance force and forced harmonic response}

Like the work in [1], the disturbance forcing is assumed as a point load normal to the surface (i.e. aligned with $\hat{e}_{r}$ ) as this loading is representative of typical experimental input, such as from a shaker or impulse hammer. Thus the disturbance force per unit area, assuming steady-state harmonic time dependence, can be written as $\tilde{\bar{F}}_{d}(x, \theta, t)=F_{d} \delta\left(x-x_{d}\right) \delta\left(\theta-\theta_{d}\right) \hat{e}_{r} \mathrm{e}^{\mathrm{j}\left(\omega t+\varphi_{d}\right)}$, where $F_{d}$ is the force amplitude. Applying this to Eq. (5) results in the following for $\tilde{\mathbf{Q}}_{d}$ :

$$
\begin{gathered}
\tilde{\mathbf{Q}}_{d}=\left\{\begin{array}{lllll}
\tilde{\mathbf{Q}}_{d}^{w \mathrm{~T}} & \mathbf{0} & \mathbf{0} & \mathbf{0} & \mathbf{0}
\end{array}\right\}^{\mathrm{T}}, \\
\tilde{\mathbf{Q}}_{d}^{w}=F_{d} \boldsymbol{\Phi}\left(x_{d}, \theta_{d}\right) \mathrm{e}^{\mathrm{j} \varphi_{d}} .
\end{gathered}
$$

As from Section 4.1, this could be extended to $N_{d}$ disturbance inputs with $\tilde{\mathbf{Q}}_{d} \in \square^{\kappa N_{s} \times N_{d}}$, though the scope of this paper is limited to $N_{d}=1$. Also, a similar scaled disturbance input matrix is defined as:

$$
\begin{gathered}
\mathbf{B}_{d}=\left[\begin{array}{lllll}
\mathbf{B}_{d}^{w \mathrm{~T}} & \mathbf{0} & \mathbf{0} & \mathbf{0} & \mathbf{0}
\end{array}\right]^{\mathrm{T}}, \\
\mathbf{B}_{d}^{w}=\left[\begin{array}{lll}
\mathbf{B}_{d}^{w, 1} & \ldots & \mathbf{B}_{d}^{w, N_{d}}
\end{array}\right], \quad \mathbf{B}_{d}^{w, r}=\frac{\tilde{\mathbf{Q}}_{d}^{w, r}}{F_{d}^{r} \mathrm{e}^{\mathrm{j} \varphi_{d}^{r}}}=\frac{\tilde{\mathbf{Q}}_{d}^{w, r}}{\tilde{F}_{d}^{r}},
\end{gathered}
$$

with $\tilde{\mathbf{Q}}_{d}=\mathbf{B}_{d} \tilde{\mathbf{u}}_{d}$ and $\tilde{\mathbf{u}}_{d}=\left\{\begin{array}{lll}\tilde{F}_{d}^{1} & \cdots & \tilde{F}_{d}^{N_{d}}\end{array}\right\}^{\mathrm{T}}$. Again, $\tilde{\mathbf{Q}}_{d}$ and $\tilde{\mathbf{Q}}_{d, o}$ are generated, as well as $\mathbf{B}_{d}$ and $\mathbf{B}_{d, o}$ via substitution of $\boldsymbol{\Phi}_{s}$ and $\boldsymbol{\Phi}_{c}$ in Eq. (5), respectively.

With the external loadings, Eq. (4) is augmented as

$$
\mathbf{M} \ddot{\mathbf{q}}+\mathbf{C} \dot{\mathbf{q}}+\tilde{\mathbf{K}} \mathbf{q}=\mathbf{B}_{d} \tilde{\mathbf{u}}_{d}+\mathbf{B}_{c} \tilde{\mathbf{u}}_{c}=\tilde{\mathbf{Q}}_{d}+\tilde{\mathbf{Q}}_{c}
$$

and assuming excitation and steady-state harmonic response (at $\omega$ ), the weighting vector $\mathbf{q}$ is:

$$
\tilde{\mathbf{q}}(\omega)=\left[\tilde{\mathbf{K}}+\mathbf{j} \omega \mathbf{C}-\omega^{2} \mathbf{M}\right]^{-1}\left\{\tilde{\mathbf{Q}}_{d}+\tilde{\mathbf{Q}}_{c}\right\}=[\tilde{\mathbf{A}}(\omega)]^{-1}\left\{\tilde{\mathbf{Q}}_{d}+\tilde{\mathbf{Q}}_{c}\right\},
$$


where $\tilde{\mathbf{A}}(\omega)$ is a reduced version (dimension $\kappa N_{s} \times \kappa N_{s}$ rather than $2 \kappa N_{s} \times 2 \kappa N_{s}$ ) of the $\tilde{\mathbf{A}}(\omega)$ matrix from the state space representation of [1]. Response vectors $\mathbf{q}_{s}$ and $\mathbf{q}_{c}$ are obtained using Eq. (15) with $\mathbf{Q}$ and $\mathbf{Q}_{o}$ in Eq. (15), and forced response spectrum in terms of out of plane motion $w(x, \theta)$ can be determined at a given frequency by Eq. (2). This result (with $V_{c}=0$ ) for an undamped shell (with dimensions from Table 1) at the response location $\left(\bar{x}_{0}, \bar{\theta}_{0}\right)=(0.98,0)$ given force excitation at $\left(\bar{x}_{d}, \bar{\theta}_{d}\right)=(0.5,0)$ was given in the recent work by Plattenburg et al. [1] for both unlined and lined shell cases. Here, overbars on locations $x$ and $\theta$ indicate normalization by $l$ and $2 \pi$, respectively, and accelerance is defined as $\tilde{A_{d}}(\omega)=\tilde{\ddot{w}}(\omega) / \tilde{F}_{d}(\omega)=-\omega^{2} \tilde{w}(\omega) / \tilde{F}_{d}(\omega)$. The forced response due to active patches can also be computed by setting $F_{d}=0$ in Eq. (12). This result is given (both unlined and lined) in Figure 5 for a patch located at $\left(\bar{x}_{c}, \bar{\theta}_{c}\right)=(0.05,0)$ and response location again at $\left(\bar{x}_{0}, \bar{\theta}_{0}\right)=(0.98,0)$. Patch properties and size are listed in Table 1. The response here is normalized in terms of acceleration per input strain from the active patch, $\tilde{A_{c}}(\omega)=\tilde{\ddot{w}}(\omega) / \tilde{\varepsilon}_{c}(\omega)$, where $\tilde{\varepsilon}_{c}=\tilde{V}_{c} d_{31}$.

\subsection{Effect of patch configurations}

Next, the shell response due to the unimorph active patch configuration (based on the simple model proposed in Section 4.1) is compared with the responses due to the in-phase (Figure $4 \mathrm{a}, M_{c x}=M_{c \theta}$ $=0$ in Eq. (10)) and out-of-phase (Figure $4 \mathrm{~b}, P_{c r}=F_{c x}=F_{c \theta}=0$ in Eq. 10) bimorph configurations. The

vibratory response transfer functions, $\tilde{A_{c}}(\omega)$, for each of the three patch configurations is plotted in Figure 6a, given patch input at $\left(\bar{x}_{c}, \bar{\theta}_{c}\right)=(0.05,0)$. Here is it seen that the response due to the unimorph configuration is dominated by the extensional input (i.e. the in-phase component) rather than the moment loading (out-of-phase component). The difference is at least an order of magnitude $(20 \mathrm{~dB})$ over the frequency range of interest; this is in agreement with Sonti and Jones [7] who showed that the in-phase bimorph configuration couple better into the lower modes than the out-of-phase configuration. 


\section{Experimental validation of model}

An experiment is designed (as shown in Figure 7) to provide evidence for the simplified formulation of the unimorph active patch configuration from Section 4. This consists of a thin shell supported by bungee cords (to simulate free boundaries) enclosed in an anechoic chamber. Since the rigid body modes associated with the shell and bungee cord system are found to be less than $10 \mathrm{~Hz}$ and sufficiently removed from the shell modes of interest, the influence of the bungee cords can be assumed negligible. Excitation is induced by an impulse hammer as well as a voltage input $\left(\tilde{V}_{c}^{r}(\omega)\right)$ to the $r$ th active patch (in the unimorph, as well as in- and out-of-phase bimorph configurations), and acceleration and sound pressure are measured at locations $\left(x_{0}, \theta_{0}\right)$ and $\left(x_{a}, \theta_{a}, r_{a}\right)$, respectively. Measurements with unimorph patch input are made with and without the presence of a passive liner of thickness $h_{L}=0.62$ mm. A strain gage is also placed on the active patch (to quantify the nature of the generic $\varepsilon_{c}=d_{31} V_{c}$ relationship).

First, the active patch strain is measured as a function of input voltage frequency (sinusoidal voltage input at constant unity gain) and the results are displayed in Figure 8. Observe here a nearly linear relationship between patch strain magnitude and excitation frequency, with approximately uniform phase. Very high coherence values above $200 \mathrm{~Hz}$ (close to $\gamma=1$ ) indicate good measurements and lend credibility to this experiment. This nearly linear relationship indicates that $d_{31}$ is of the following

empirical form: $\tilde{d}_{31}(\omega) \approx \omega\left|d_{31}\right| \mathrm{e}^{\mathrm{j} \phi_{c}}$. Alternately, this could be interpreted as the output strain of the active patch being proportional to the current through the patch. Indeed, if the active patch is assumed to be a purely capacitive element, the AC current-voltage relationship is of the form:

$$
\tilde{V}(\omega)=\frac{1}{\mathrm{j} \omega C} \tilde{I}(\omega)
$$

Thus, with uniform voltage, the corresponding current would be linear with frequency and have a constant phase angle of $+90^{\circ}$, nearly identical to the strain plots from Figure 8. Based on a linear regression of measured data, the observed proportionality constant between frequency and control strain 
is approximately $5.4 \times 10^{-4} \mu \varepsilon-\mathrm{V}^{-1}-\mathrm{Hz}^{-1}$ in the principal patch direction and $3.4 \times 10^{-4} \mu \varepsilon-\mathrm{V}^{-1}-\mathrm{Hz}^{-1}$ for the orthogonal direction. These experimental measurements, having high coherence, indicate validity of this linear relationship for this particular configuration. Therefore the observed empirical transfer function $\left(\frac{\tilde{\varepsilon}_{c}}{\tilde{V}_{c}}(\omega)=\tilde{d}_{31}(\omega)\right)$ will be used to map a measured $\frac{\tilde{\ddot{w}}}{\tilde{V}_{c}}(\omega)$ transfer function to the desired $\tilde{A_{c}}(\omega)=\frac{\tilde{\tilde{w}}}{\tilde{\varepsilon}_{c}}(\omega)$ (for the sake of comparison with the model).

Next, acceleration and sound pressure measurements are made subject to active patch input. The acceleration magnitude results (normalized by input strain) are shown in in Figure 6 for all three active patch configurations. There is reasonable agreement between the experiment (Fig. 6b) and the model (Fig. 6a), especially in terms of the qualitative shape of the magnitude spectra (i.e. relative magnitudes among three configurations, and relative locations of peaks and valleys). The absolute magnitudes of the

measured $\tilde{A_{c}}(\omega)$ spectra deviate from the analytical results by a substantial amount (approximately 30 $\mathrm{dB}$ ), primarily due to the unmodeled dynamics of the piezoelectric patch, and the simplified piezoelectric relationships assumed [7,9]. While a more comprehensive model relaxing some of these simplifying assumptions could certainly be developed in the future, as a first approximation the proposed analytical formulation is deemed sufficient for comparative studies among different configurations and evaluating relative vibratory attenuation effects. Next, the approximate model developed in Section 4 will be used to design a vibration control system.

\section{Design of alternate control schemes for steady-state harmonic response}

\subsection{Design for local or global control}

If there are critical components at certain isolated locations (e.g. at $\left.\left(x_{0}, \theta_{0}\right)\right)$, it may be desired to force the transverse motion $w_{0}=w\left(x_{0}, \theta_{0}\right)$ to vanish. Assuming harmonic excitation and steady state response, Eq. (15) is used to compute q. Then, setting Eq. (2) equal to zero, 
$w(x, \theta)=\left[\mathbf{q}_{s}^{w}\right]^{\mathrm{T}} \mathbf{\Phi}_{s}^{w}(x, \theta)+\left[\mathbf{q}_{c}^{w}\right]^{\mathrm{T}} \boldsymbol{\Phi}_{c}^{w}(x, \theta)=0$, and substituting the result from Eq. (15) for $\mathbf{q}$, the complex-valued control effort $\tilde{V}_{c}$ required to achieve this condition (with $N_{c}=1$ ) is given by:

$$
\tilde{V}_{c}(\omega)=-\frac{\left\{\mathbf{B}_{d}[\tilde{\mathbf{A}}(\omega)]^{-1}\right\}^{\mathrm{T}} \boldsymbol{\Phi}_{s}^{w}\left(x_{0}, \theta_{0}\right)+\left\{\mathbf{B}_{d, o}[\tilde{\mathbf{A}}(\omega)]^{-1}\right\}^{\mathrm{T}} \boldsymbol{\Phi}_{c}^{w}\left(x_{0}, \theta_{0}\right)}{\left\{\mathbf{B}_{c}[\tilde{\mathbf{A}}(\omega)]^{-1}\right\}^{\mathrm{T}} \boldsymbol{\Phi}_{s}^{w}\left(x_{0}, \theta_{0}\right)+\left\{\mathbf{B}_{c, o}[\tilde{\mathbf{A}}(\omega)]^{-1}\right\}^{\mathrm{T}} \boldsymbol{\Phi}_{c}^{w}\left(x_{0}, \theta_{0}\right)} \tilde{F}_{d} .
$$

Note here (and throughout Section 6) that only the first $N_{s}$ element of the vectors $\left\{\tilde{\mathbf{B}}_{d}[\tilde{\mathbf{A}}(\omega)]^{-1}\right\}^{\mathrm{T}}$ and $\left\{\tilde{\mathbf{B}}_{c}[\tilde{\mathbf{A}}(\omega)]^{-1}\right\}^{\mathrm{T}}$ are retained so the multiplication with $\boldsymbol{\Phi}^{w}$ is well-defined. If multiple response locations are desired to be controlled (i.e. $\left(x_{0}{ }^{i}, \theta_{0}{ }^{i}\right)$ for $\left.i=1, \ldots, N_{0}\right)$ a system of $N_{0}$ such equations must be defined. Given $N_{c}$ control inputs, a matrix equation is defined as:

$$
\begin{gathered}
\tilde{\mathbf{D}}(\omega) \tilde{\mathbf{u}}_{c}=\tilde{\mathbf{d}}, \\
\tilde{D}_{i j}=\left\{[\tilde{\mathbf{A}}(\omega)]^{-1} \tilde{\mathbf{B}}_{c}^{j}\right\}^{\mathrm{T}} \boldsymbol{\Phi}_{s}^{w}\left(x_{0}^{i}, \theta_{0}^{i}\right)+\left\{[\tilde{\mathbf{A}}(\omega)]^{-1} \tilde{\mathbf{B}}_{c, o}^{j}\right\}^{\mathrm{T}} \boldsymbol{\Phi}_{c}^{w}\left(x_{0}^{i}, \theta_{0}^{i}\right), \\
\tilde{d}_{i}=-\left[\left\{[\tilde{\mathbf{A}}(\omega)]^{-1} \tilde{\mathbf{B}}_{d}\right\}^{\mathrm{T}} \boldsymbol{\Phi}_{s}^{w}\left(x_{0}^{i}, \theta_{0}^{i}\right)+\left\{[\tilde{\mathbf{A}}(\omega)]^{-1} \tilde{\mathbf{B}}_{d, o}\right\}^{\mathrm{T}} \boldsymbol{\Phi}_{c}^{w}\left(x_{0}^{i}, \theta_{0}^{i}\right)\right] \tilde{F}_{d},
\end{gathered}
$$

where $\mathbf{B}_{c}^{j}$ is the $j$ th column of matrix $\mathbf{B}_{c}$. This system can be solved for the vector of complex control efforts, $\tilde{\mathbf{u}}_{c}$, as a function of frequency if $\tilde{\mathbf{D}}$ is invertible, which from control theory requires that the $N_{0^{-}}$ dimensional observable subspace and the $N_{c}$-dimensional controllable subspace have equivalent span [31].

Alternately, some applications or design constraints require a minimization of global vibration or acoustic radiated sound pressure, since the sound power of the radiating source, $W_{p}$, is proportional to its mean-square transverse surface velocity, $\|\dot{w}(x, y ; \omega)\|^{2}$ [32]. Here $\|\cdot\|$ indicates the surface averaged root mean-square (RMS) value. Based on the derivation of the equations of motion from [1] and Sections 3-4, the global vibration is expressed in terms of the surface-averaged mean-square value of shell displacement as a quadratic function of $\mathbf{q}$ : 


$$
\|w\|^{2}(\omega)=\iint_{A}\left[\left\{\tilde{\mathbf{q}}_{s}^{w}\right\}^{\mathrm{T}} \boldsymbol{\Phi}_{s}^{w}+\left\{\tilde{\mathbf{q}}_{c}^{w}\right\}^{\mathrm{T}} \boldsymbol{\Phi}_{c}^{w}\right]\left[\left\{\tilde{\mathbf{q}}_{s}^{w}\right\}^{\mathrm{T}} \boldsymbol{\Phi}_{s}^{w}+\left\{\tilde{\mathbf{q}}_{c}^{w}\right\}^{\mathrm{T}} \boldsymbol{\Phi}_{c}^{w}\right]^{\mathrm{H}} d A
$$

where superscript $\mathrm{H}$ indicates the Hermitian transpose. The displacement $\|w\|^{2}(\omega)=\Psi_{w}^{2}(\omega)$ can then be related to the mean-square velocity, $\Psi_{\dot{w}}^{2}$, by a factor of $\omega^{2}$. A sound pressure reduction is then equivalent to a reduction in sound power or surface velocity (at $\omega$ ) in terms of the insertion loss (IL, e.g. $\mathrm{IL}_{w}$ for vibratory, $\mathrm{IL}_{p}$ for acoustic, etc.), defined as: $I L=10 \log _{10}\left(\Psi_{u n t .}^{2} / \Psi_{t .}^{2}\right)[\mathrm{dB}]$. Here $\Psi^{2}$ is the mean-square value of some response (i.e. sound pressure, surface velocity, etc.) and subscripts $t$. and $u n t$. refer to treated and untreated, respectively.

To minimize the global vibration $\Psi_{w}^{2}$, Eq. 19 is differentiated with respect to the complex-valued control effort $\tilde{V}_{c}$. Again, if multiple control efforts $\tilde{V}_{c}^{i}$ are used (with $i=1, \ldots, N_{c}$ ), this generalizes readily by using partial derivatives of $\Psi_{w}^{2}$ with respect to the $N_{c}$ control efforts. This results in $N_{c}$ decoupled equations for the $i$ th control effort as:

$$
\begin{aligned}
\tilde{V}_{c}^{i}(\omega)=-\frac{\left.\left\{[\tilde{\mathbf{A}}(\omega)]^{-1} \mathbf{B}_{d}\right\}^{\mathrm{H}} \mathbf{S}\left\{[\tilde{\mathbf{A}}(\omega)]^{-1} \mathbf{B}_{c}^{i}\right\}+\left\{[\tilde{\mathbf{A}}(\omega)]^{-1} \mathbf{B}_{d, o}\right\}\right\}^{\mathrm{H}} \mathbf{S}\left\{[\tilde{\mathbf{A}}(\omega)]^{-1} \mathbf{B}_{c, o}^{i}\right\}}{\left\{[\tilde{\mathbf{A}}(\omega)]^{-1} \mathbf{B}_{c}^{i}\right\}^{\mathrm{H}} \mathbf{S}\left\{[\tilde{\mathbf{A}}(\omega)]^{-1} \mathbf{B}_{c}^{i}\right\}+\left\{[\tilde{\mathbf{A}}(\omega)]^{-1} \mathbf{B}_{c, o}^{i}\right\}^{\mathrm{H}} \mathbf{S}\left\{[\tilde{\mathbf{A}}(\omega)]^{-1} \mathbf{B}_{c, o}^{i}\right\}} \tilde{F}_{d}, \\
\mathbf{S}=a \int_{0}^{2 \pi} \int_{0}^{l}\left\{\boldsymbol{\Phi}_{s}^{w}(x, \theta)\right\}\left\{\boldsymbol{\Phi}_{s}^{w}(x, \theta)\right\}^{\mathrm{T}} d x d \theta=a \int_{0}^{2 \pi} \int_{0}^{l}\left\{\boldsymbol{\Phi}_{c}^{w}(x, \theta)\right\}\left\{\boldsymbol{\Phi}_{c}^{w}(x, \theta)\right\}^{\mathrm{T}} d x d \theta
\end{aligned}
$$

where $\mathbf{S}$ is a constant matrix of the integral of the shape functions. The cross terms associated with $\boldsymbol{\Phi}_{s} \boldsymbol{\Phi}_{c}$ fortuitously drop out due to their orthogonality, greatly simplifying the expression in Eq. (20b). Again, only the first $N_{s}$ elements of the vectors $\left\{\mathbf{B}[\tilde{\mathbf{A}}(\omega)]^{-1}\right\}^{\mathrm{T}}$ are retained here. Note that for uniform thickness and density shells, $\rho h \mathbf{S}=\mathbf{M}_{11}$ (in the notation of [1]), and $\mathbf{S}$ is a constant diagonal matrix if the shape functions are mutually orthogonal (which is approximately satisfied for free boundaries). In this case, the numerator and denominator become inner products between vectors $\left\{\mathbf{B}[\tilde{\mathbf{A}}(\omega)]^{-1}\right\}^{\mathrm{T}}$ and then $\tilde{V}_{c}^{i}$ in Eq. 
(20a) can be interpreted as the projection of the disturbance vector onto the $i$ th control input vector (with negative direction) in the $N_{s}$-dimensional shape function space.

\subsection{Effect of control scheme design}

In order to verify the derivations of Section 6.1, a brief study is given here. First, the value of local response, $w_{0}=w\left(x_{0}, \theta_{0}\right)$, is checked under local control scheme of Eqs. (17) and (18). The resulting operating displacement shape of the shell is computed with and without control for an active patch at location 1 (i.e. from Table 1$)$, measurement location at $\left(\bar{x}_{0}, \bar{\theta}_{0}\right)=(0.98,0.0)$, and excitation at the $5^{\text {th }}$ natural frequency, corresponding to the $(2,3)$ mode. The result is plotted in Figure 9, where $|\bar{w}|$ denotes absolute value and normalization by the maximum value of the uncontrolled response (thus Figure 9a has range $0 \leq|\bar{w}| \leq 1)$. Observe that $|\bar{w}|$ is indeed forced to 0 at the measurement location under the local control scheme, and furthermore, the overall response is reduced by approximately $95 \%$. The control effort effectively rotates the mode shape in the $\theta$ direction, forcing $|\bar{w}|$ at the response location $\left(x_{0}, \theta_{0}\right)$ from nearly a maximum to zero.

Next, the global control scheme of Eq. (20) is verified, keeping most parameters the same as in the local control study of Figure 9 (disturbance/patch location and excitation frequency). The shell response due to the optimal value of $\tilde{V}_{c}$ is compared in Figure 10a and $10 \mathrm{~b}$ with other values of the control effort—ranging in magnitude from $0.1\left|\tilde{V}_{c}\right|$ to $10\left|\tilde{V}_{c}\right|$ and in phase from $\angle \tilde{V}_{c} \pm \pi$, respectively. Here the global response is computed for each value of $\tilde{V}_{c}$, and normalized such that $\Psi_{w}^{2}\left(\tilde{V}_{c, o p t}\right)=0 \mathrm{~dB}$.

The displacement profile for the optimal value of $\tilde{V}_{c}$ is also given in Figure 10c. Note from Figures 10a and $\mathrm{b}$ that the minimum value of $\Psi_{w}^{2}$ in both cases is coincident with the optimal value of $\tilde{V}_{c}$ as computed from Eq. (20). It can be further observed from Figure 10c that the displacement profile is rather similar to that under local control in Figure 10b; indeed in all cases the $(2,3)$ mode is still the dominant participant. However, under global control $\Psi_{w}^{2}$ is reduced by a finite, albeit marginal, value of $0.2 \mathrm{~dB}$ 
relative to local control, consistent with the hypothesis that global control should give the smallest value of $\Psi_{w}^{2}$ for any control scheme.

Finally, the control transfer functions $\tilde{\Xi}(\omega)=\tilde{V}_{c}(\omega) / \tilde{F}_{d}(\omega)$ are compared in Figure 11 for both the local and global control schemes (with measurement location $\left(\bar{x}_{0}, \bar{\theta}_{0}\right)=(0.98,0.0)$, disturbance location $\left.\left(\bar{x}_{d}, \bar{\theta}_{d}\right)=(0.56,0.0)\right)$, and two active patch locations (from Table 1). The required control effort is a function of frequency, active patch location, and control scheme. The value of $\Xi$ tends to take on a higher value at resonant modes, which intuitively suggests that more energy is needed to control the modal response. It is also seen that patch location 1 typically results in a somewhat smaller value of $\Xi$ independent of the control scheme, meaning that it would be a "cheaper" location, to say nothing of the resulting value of $\|w\|^{2}$ or $w_{0}$. Since the global control scheme is most applicable for acoustic radiation problems, this scheme will be used for the remainder of the paper.

\section{Vibration control performance metrics}

In order to evaluate the broadband shell response, first, consider the total kinetic energy associated with transverse motion of a uniform shell, $T(\omega)=\frac{1}{2} \rho h A \Psi_{\dot{w}}^{2}(\omega)$, where $A$ is the surface area of the shell. Then, define a "pseudo-mechanical power" to be the temporal derivative of $T$, namely $W_{m}(\omega)$ $=\omega T$ for harmonic motion, having units of Watts. This power value captures the time-averaged vibratory energy of the structure. Next define the broadband attenuation of the mechanical power, $W_{m}$, to be:

$$
\Delta \underline{W}_{m}=\underline{W}_{m}^{u n c .}-\underline{W}_{m}^{c .}=\frac{1}{\Omega_{2}-\Omega_{1}} \int_{\Omega_{1}}^{\Omega_{2}} W_{m}^{u n c .}(\omega) d \omega-\frac{1}{\Omega_{2}-\Omega_{1}} \int_{\Omega_{1}}^{\Omega_{2}} W_{m}^{c .}(\omega) d \omega .
$$

Here, underbars denote integrated quantities, superscripts $c$. and unc. refer to controlled and uncontrolled, respectively, and $\Omega_{1}$ and $\Omega_{2}$ are the lower and upper frequency limits. Thus, a large value of $\Delta \underline{W}_{m}$ corresponds to an effective vibration control system. This representation may provide an alternate means of quantifying attenuation, as opposed to insertion loss at a single frequency, because the presence of the 
cardboard liner may result in shifting of natural frequencies (as discussed in [1]). Next, consider the input electrical power (units of $[\mathrm{W}]$ ) to the active patches, defined as:

$$
W_{c}(\omega)=\frac{1}{2} \tilde{V}_{c} \tilde{I}_{c}^{*}=\frac{1}{2} \omega C\left|\tilde{V}_{c}(\omega)\right|^{2}, \quad \underline{W}_{c}=\frac{1}{\Omega_{2}-\Omega_{1}} \int_{\Omega_{1}}^{\Omega_{2}} W_{c}(\omega) d \omega
$$

where $I$ is the input current in the frequency domain and $C$ is the capacitance of the active patch (nominally $1 \mathrm{nF}$ for the MFC patches of this research [21]). Ideally, this input electrical power would be small for an efficient control system design. Finally, two normalized performance metrics (both dimensionless) are defined as follows:

$$
\Pi_{1}=\frac{\Delta \underline{W}_{m}}{\underline{W}_{m}^{u n c .}}=\frac{\underline{W}_{m}^{u n c .}-\underline{W}_{m}^{c .}}{\underline{W}_{m}^{u n c .}}, \quad \quad \Pi_{2}=\frac{\Delta \underline{W}_{m}}{\Delta \underline{W}_{m}+\alpha \underline{W}_{c}} .
$$

Here, $\Pi_{1}$ quantifies strictly performance in terms of reduction of vibratory energy, and ranges from $0 \%$ (no reduction) to $100 \%$ (full vibration attenuation). The metric $\Pi_{2}$, on the other hand represents "efficiency" of the control system; it ranges from 0 to 1 , and has a value close to 1 when attenuation is high while input power is not too large. Because the extreme value of 1 will never truly be reached with finite input, the absolute value of $\Pi_{2}$ is less important than relative values in a comparative study. Thus, the empirical factor $\alpha$ is simply a conditioning parameter to keep $\Pi_{2}$ from becoming very close to 0 or 1 and to facilitate comparisons if the relative values of $\Delta \underline{W}_{m}$ and $\underline{W}_{c}$ become very disparate.

\section{Illustrative vibration control design studies}

\subsection{Selection of active patch candidate locations}

Several active patch locations are considered, with either one or two patches, to determine the resulting shell response and control input for the global control scheme. This study is limited to the locations $\left(x_{0}, \theta_{0}\right)$ and $\left(x_{d}, \theta_{d}\right)$ given previously (from Table 1), and the shell modes in the range 200-2000 Hz. Three patch locations- $\left(\bar{x}_{c}, \bar{\theta}_{c}\right)$-are considered here as follows (also shown in Figure 12): (1) a single patch at $(0.05,0.0)$; (2) a single patch at $(0.5,0.0)$; and (3) two patches at $(0.05,0.0)$ and $(0.5,0.0)$. These locations are chosen to attack $\theta$-type modes (1) and $m=$ even $x$-type modes (2), or both (3). 
To compare the three locations, the broadband global mean-square velocity $\left(\Psi_{w}^{2}(\omega)=\omega^{2}\|w\|^{2}\right)$ is computed for each case under the global control scheme, and the results are plotted in Figure 13. It is seen here that location 2 seems to provide better control in the broadband sense than location 1 . However, at several of the modes, in particular those with odd $m$, location 2 is less suited for control. Location 3 appears to provide the best performance in all cases (as might be expected, since more energy is consumed from using two patches).

\subsection{Simulation of comparative designs}

Next, the following vibration control designs are numerically evaluated (as illustrated in Figure 14): (I) a passive liner (i.e. the case of [1]); (II) one active patch at location 2; (III) one active patch at location 2 along with a passive liner. A single liner thickness of $h_{L}=0.62 \mathrm{~mm}$ is used; interfacial damping is assumed to be $c_{v}=1 \times 10^{5}$ (a typical value from [1]); 200-2000 Hz is taken for typical acoustic applications; and patch location 2 is selected based on the insights gained from Section 8.1. While two active patches may provide superior attenuation, the relative effects of active patch vs. passive liner are of more interest here. For each case, the global responses are computed along with metrics $\Pi_{1}$ (performance) and $\Pi_{2}$ (efficiency, with $\alpha=100$ ), given in Table $3 \mathrm{a}$. The IL values (of global velocity) for each case relative to the unlined shell are also listed for selected modes in Table 3b, representing single frequency attenuation. From Table 3 it is seen that theoretically, while an active patch can provide large attenuation for some modes, the location of a particular patch may limit the IL at other modes (e.g. mode 4). The addition of the passive damping liner should improve vibratory reduction in terms of both $\Pi_{1}$ and $\Pi_{2}$ values, indicating improvement in both performance and efficiency. Furthermore, the concurrent damping should enhance the modal IL value of the active-only case (design II) and improve the attenuation in the broadband sense. This suggests that the concurrent active/passive approach could be beneficial for many applications. 


\subsection{Experimental study for a single mode}

Finally, as an illustrative case study, the effects of alternate treatments-designs I, II, and III of Section 8.2 -are experimentally studied at the $(2,3)$ mode. An electrodynamic shaker is attached to the freely suspended shell at $\left(\bar{x}_{0}, \bar{\theta}_{0}\right)=(0.55,0.6)$ via a compliant stinger (see Figure 7$)$ and used to excite the shell at a single frequency close to the $(2,3)$ mode. The input force is also measured via an interfacial load cell. Note that the voltage input to the shaker (disturbance) is selected in each case to ensure equal force input $\left(F_{d}=0.016 \mathrm{~N}_{\mathrm{RMS}}\right)$. The active patch is then used to attenuate both local acceleration at $\left(\bar{x}_{0}, \bar{\theta}_{0}\right)$ $=(0.0,0.98)$ as well as sound pressure at $\left(\bar{x}_{a}, \bar{\theta}_{a}, \bar{r}_{a}\right)=(0.5,0.1,0.5)$. This is achieved by selecting the voltage magnitude and phase input to the active patch such that the response is equal to (and out of phase from) that of the disturbance, termed as "user-in-the-loop" control. A summary of the experiments, including the excitation frequency for unlined and lined cases (993 and $968 \mathrm{~Hz}$, respectively), the response magnitudes for both acceleration and sound pressure level (SPL), and the insertion loss on a dB basis due to the treatments are given in Table 4.

The following observations are made from the measurements (Table 4) which are qualitatively consistent with the predictions summarized in Table 3. First, the passive liner induces modest IL for both cases, similar to the experiments of [1]. The use of active patches greatly increases this IL value to 40 to $50 \mathrm{~dB}$. Second, when active patches are used concurrently with the passive liner, it is seen that the IL values are now similar to that of the active-only case. However, it should be noted that the "user-in-theloop" method (in this article) is subject to more variation a feedback control system, and active control may depend on maintaining very precise control parameters. Third, the inclusion of the passive liner induces additional broadband attenuation as a further benefit [1]. As a final observation, note that both the control input magnitude and phase are different for the lined vs. unlined case, as well as for control of acceleration (analogous to the local control scheme) and sound pressure (global control scheme). This reflects the differences in the transfer functions of Figures 5 and 11 between unlined/lined cases and local/global schemes. 


\section{Conclusion}

This paper extends the recent article by Plattenburg et al. [1] by proposing a refined semianalytical model and presenting model-based design studies for a thin cylindrical shell with concurrent active piezoelectric patches and a distributed cardboard damping liner. The concurrent active/passive approach is selected due to a lack of prior analytical studies in this area, as well as applicability to modern lightweight vehicles with noise sources in the middle and higher frequency ranges [2-6]. The model developed here is an extension of the prior shell formulation [1] and takes into account the effects of active patches in a novel configuration (unimorph), extending the work of Sonti and Jones [7] and Chaudhry et al. [9]. Experiments are performed on a shell with active patches (under harmonic excitation from 200 to $2000 \mathrm{~Hz}$ ) in several patch configurations to provide evidence for the analytical model predictions. Alternate control schemes are derived and given in terms of transfer functions, and two energy-based performance metrics are suggested which facilitate the design study by allowing direct comparison of alternate designs. Finally, passive, active, and concurrent approaches are considered in a comparative study (both analytically and experimentally). The results of this study indicate that additional active patches may provide significant enhancement of vibratory attenuation but with potentially larger associated cost, whereas inclusion of a distributed liner should provide broadband benefits for many modes simultaneously.

The proposed model captures the dynamics of shell structures (with specific application to the contemporary automotive industry, especially its need for lightweight noise and vibration control treatments) and allows for a scientific comparison of competing vibration control strategies, while providing valuable insight. Also, results illustrate the utility of the proposed model for design of other practical systems in terms of minimizing vibro-acoustic response of such systems. Further refinements to the research could include improving the active patch model by considering its mass/stiffness distribution or electromechanical dynamics, and modeling the finite bonding layer thickness. Furthermore, a real-time feedback control system could be included to improve the fidelity of the experimental results. 


\section{Acknowledgements}

We acknowledge the OSU Graduate School, the Ohio Space Grant Consortium, the Smart Vehicle Concepts Center (www.SmartVehicleCenter.org), and the National Science Foundation

Industry/University Cooperative Research Centers program (www.nsf.gov/eng/iip/iucrc) for their financial support of this research. 


\section{References}

[1] J. Plattenburg, J. T. Dreyer, R. Singh, New analytical model for vibration of a cylindrical shell and cardboard liner with focus on interfacial distributed damping, Mechanical Systems and Signal Processing, 75 (2016) 176-195.

[2] Z. Sun, D. Schankin, W. Braun, and J. Ley, Attenuation of driveline vibrations through tuning of propeller shaft liners, SAE Technical Paper 2011-01-1547, SAE Noise and Vibration Conference, 2011.

[3] M.G. Foulkes, J. P. DeClerck, and R. Singh, Vibration characteristics of cardboard inserts in shells, SAE Transactions Journal of Passenger Cars - Mechechanical Systems, 112 (6) (2004) 1718-1725.

[4] S. Thoedossiades, H. Rahnejat, and P. Kelly, On the effect of cardboard liners on impact-induced high frequency vehicular driveline vibrations, ASME Paper No. DETC2007-35692, 2007.

[5] M. Gnanakumarr, P. D. King, S. Theodossiades, and H. Rahnejat, Methods of palliation for high frequency elasto-acoustic response of truck drivetrain systems, International Journal of Heavy Vehicle Systems, 13 (4) (2006) 253-262.

[6] H. Koruk, J. T. Dreyer, and R. Singh, Modal analysis of thin cylindrical shells with cardboard liners and estimation of loss factors, Mechanical Systems and Signal Processing, 45 (2014) 346-359.

[7] V. R. Sonti and J. D. Jones, Curved piezoactuator model for active vibration control of cylindrical shells, AIAA Journal, 34 (5) (1996) 1034-1040.

[8] V. R. Sonti and J. D. Jones, Dynamic effects of piezoactuators on the cylindrical shell response, AIAA Journal, 34 (4) (1996) 795-801

[9] Z. Chaudhry, F. Lalande, and C. A. Rogers, Modeling of induced strain actuation of shell structures, Journal of the Acoustical Society of America, 97 (5) (1995) 2872-2877.

[10] C. R. Fuller, C. H. Hansen, and S. D. Snyder, Active control of sound radiation from a vibrating rectangular panel by sound sources and vibration inputs: an experimental comparison, Journal of Sound and Vibration, 145 (2) (1991) 195-215.

[11] J. H. Han and I. Lee, Optimal placement of piezoelectric sensors and actuators for vibration control of a composite plate using genetic algorithms, Smart Materials and Structures, 8 (2) (1999), 257267.

[12] T. Bailey and J. E. Hubbard, Distributed piezoelectric-polymer active vibration control of a cantilever beam, Journal of Guidance, Control, and Dynamics, 8 (5) (1985) 605-611.

[13] R. H. Cabell, C. R. Fuller, A principal component algorithm for feedforward active noise and vibration control, Journal of Sound and Vibration, 227 (1) (1999) 159-181.

[14] M. K. Kwak, S. Heo, and M. Jeong, Dynamic modelling and active vibration controller design for a cylindrical shell equipped with piezoelectric sensors and actuators, Journal of Sound and Vibration, 321 (2009) 510-524.

[15] A. Benjeddou, Advances in hybrid active-passive vibration and noise control via piezoelectric and viscoelastic constrained layer treatments, Journal of Vibration and Control, 7 (2001) 565-602. 
[16] A. Baz and J. Ro, Optimum design and control of active constrained layer damping, ASME Journal of Mechanical Design, 117 (B) (1996) 135-144.

[17] A. Baz and J. Ro, Vibration control of plates with active constrained layer damping, Smart Materials and Structures, 5 (1996) 272-280.

[18] M. C. Ray, J. Oh, and A. Baz, Active constrained layer damping of thin cylindrical shells, Journal of Sound and Vibration, 240 (5) (2001) 921-935.

[19] M. J. Lam, D. J. Inman, and W. R. Saunders, Vibration control though passive constrained layer damping and active control, Journal of Intelligent Material Systems and Structures, 8 (1997) 663677.

[20] J. Plattenburg, J. T. Dreyer, and R. Singh, Active and passive damping patches on a thin rectangular plate: A refined analytical model with experimental validation, Journal of Sound and Vibration, 353 (2015) 75-95.

[21] H. Zheng, C. Cai, G. S. H. Pau, and G. R. Liu, Minimizing vibration response of cylindrical shells through layout optimization of passive constrained layer damping treatments, Journal of Sound and Vibration, 279 (2005) 739-756.

[22] L. H. Chen and S. C. Huang, Vibrations of a cylindrical shell with partially constrained layer damping (CLD) treatment, International Journal of Mechanical Sciences, 41 (1999), 1485-1498.

[23] H. J. Wang and L. W. Chen, Finite element dynamic analysis of orthotropic cylindrical shells with a constrained damping layer, Finite Elements in Analysis and Design, 40 (7) (2004) 737-755.

[24] MFC Actuator by Smart Material Corp, 〈http://www.smart-material.com/MFC-product-main.html〉 (accessed Jan. 4, 2016).

[25] C. B. Sharma, Calculation of natural frequencies of fixed-free circular cylindrical shells, Journal of Sound and Vibration, 35 (1) (1974) 55-76.

[26] W. Soedel, Vibration of Plates and Shells, Marcel Dekker, New York, 2004.

[27] A. Leissa, Vibration of Shells, Acoustical Society of America, 1993.

[28] L. Meirovitch, Fundamentals of Vibrations, McGraw-Hill, New York, 2001

[29] E. K. Dimitriadis, C. R. Fuller, and C. A. Rogers, Piezoelectric actuators for distributed vibration excitation of thin plates, Journal of Vibration and Acoustics, 113 (1991) 100-107.

[30] E. F. Crawley and E. H. Anderson, Detailed models of piezoceramic actuation of beams, Journal of Intelligent Material Systems and Structures, 1 (1) (1990), 4-25.

[31] P. J. Antsaklis and A. N. Michel, A Linear Systems Primer, Birkhauser, Boston, MA, 2007.

[32] C. Wang and J. C. S. Lai, The sound radiation efficiency of finite length acoustically thick circular cylindrical shells under mechanical excitation I: theoretical analysis, Journal of Sound and Vibration, 232 (2) (2000), 431-447. 


\section{Appendix A: List of Symbols}

\begin{tabular}{|c|c|}
\hline \multicolumn{2}{|c|}{ Nomenclature } \\
\hline $\bar{a}$ & $\overline{\text { radius }}$ \\
\hline$A$ & surface area (for integration) \\
\hline $\mathbf{A}$ & system matrix \\
\hline$A$ & accelerance \\
\hline B & input matrix \\
\hline$c$ & viscous friction coefficient \\
\hline $\mathbf{C}$ & viscous damping matrix \\
\hline$d_{i j}$ & piezoelectric coefficient \\
\hline d & control-to-disturbance vector \\
\hline D & control-to-disturbance matrix \\
\hline $\mathrm{e}$ & exponential constant \\
\hline E & Young's modulus \\
\hline$f$ & frequency $[\mathrm{Hz}]$ \\
\hline$F$ & force \\
\hline$h$ & layer thickness \\
\hline$I$ & electric current \\
\hline $\mathrm{j}$ & imaginary number, $\sqrt{-1}$ \\
\hline K & stiffness matrix \\
\hline$l$ & length \\
\hline$M$ & moment \\
\hline $\mathbf{M}$ & mass matrix \\
\hline$N$ & number (patches, shape functions, etc.) \\
\hline$p$ & sound pressure \\
\hline $\mathbf{Q}$ & generalized force vector \\
\hline $\mathbf{q}$ & shape function weighting vector \\
\hline$r$ & radial coordinate \\
\hline$t$ & time \\
\hline$T$ & kinetic energy \\
\hline$u, v$ & lateral displacements (in $x, \theta$ direction) \\
\hline $\mathbf{u}$ & input vector (control or disturbance) \\
\hline$U$ & potential (strain) energy \\
\hline$V$ & voltage \\
\hline$w$ & transverse displacement \\
\hline$W$ & power (mechanical, electric, etc.) \\
\hline$x$ & axial coordinate, endpoint, or location \\
\hline$X$ & axial shape function \\
\hline$\alpha$ & $\begin{array}{l}\text { conditioning parameter for performance } \\
\text { metric }\end{array}$ \\
\hline$\gamma$ & coherence \\
\hline$\Delta$ & change or shift in value \\
\hline$\varepsilon$ & normal strain \\
\hline$\eta$ & loss factor \\
\hline$\theta$ & $\begin{array}{l}\text { circumferential coordinate, endpoint, or } \\
\text { location }\end{array}$ \\
\hline$\Theta$ & circumferential shape function \\
\hline
\end{tabular}

$\kappa \quad$ order of problem (full or reduced)

$v \quad$ Poisson's ratio

$\Xi \quad$ control transfer function

$\Pi \quad$ performance metric

$\rho \quad$ mass density per unit volume

$\phi \quad$ Rayleigh-Ritz shape function

$\varphi \quad$ phase between disturbance and control

Ф shape function vector

$\psi^{2} \quad$ mean-square value

$\omega \quad$ angular frequency [ $\mathrm{rad} / \mathrm{s}]$

\section{Subscripts}

$\begin{array}{ll}0 & \text { motion location } \\ a & \text { acoustic measurement location }\end{array}$

$c$ piezoelectric (control) layer or input

$d$ disturbance input

$i \quad$ counting index for layers and modes

$k \quad$ counting index for shape functions

$L \quad$ cardboard liner

$m \quad$ modal index (in $x$ direction)

$n \quad$ modal index (in $\theta$ direction)

$o$ orthogonal shape function set

$s \quad$ shape function

- $\quad$ integrated quantity (underbar)

\section{Superscripts}

$\alpha \quad$ arbitrary motion type (transverse, etc.)

$u, v, w$ shape functions for different motions

$\sim \quad$ complex valued

- normalized or dimensionless (overbar)

$\rightarrow \quad$ vector (in space)

* complex conjugate

$\mathrm{T} \quad$ standard matrix transpose

$\mathrm{H} \quad$ Hermitian transpose

\section{Operators}

|. $\quad$ absolute value or magnitude (for complex number)

$\|\cdot\| \quad$ surface-averaged RMS value

$\angle$ argument (angle) of complex number

$H \quad$ Heaviside (unit step) function

$\delta \quad$ Dirac delta (unit impulse) function

$\delta^{\prime} \quad$ derivative of Dirac delta function

\section{Abbreviations}

IL insertion loss

RMS root mean square

SPL sound pressure level 


\section{List of Figure Captions}

Figure 1: Example case: active patches and cardboard liner on a thin cylindrical shell with free boundaries (extension of the system studied in [1]); patch size/location and force/measurement location are labeled; see Appendix A and text for definition of symbols

Figure 2: Schematics of shell sector (in the $\theta-r$ plane) with three configurations: (a) bimorph with in-phase actuation; (b) bimorph with out-of-phase actuation; (c) unimorph with cardboard liner

Figure 3: Effective distributed pressure loading: (a) in-phase actuation; (b) equivalent force loading; (c) resultant of equivalent force $(\leftarrow)$ needs to be balanced by an effective pressure $(--->)$

Figure 4: Equivalent loading from alternate active patch configurations on a shell section: (a) in-phase bimorph with $F_{x}, F_{\theta}$, and $P_{r}(\rightarrow)$ and patch endpoints labeled; (b) out-of-phase bimorph with $M_{x}$ and $M_{\theta}(---)$ ); (c) proposed combined loading representation of unimorph

Figure 5: Effect of control input from an active patch on predicted vibratory response spectra of a thin shell $\left(\right.$ i.e. $\left.A_{c}(\omega)\right)$, unlined $(-)$, and lined $(\cdots)$, with modal values denoted by large dots $(\bullet)$

Figure 6: Comparison of unimorph and bimorph responses, in terms of $\tilde{A_{c}}(\omega)$ magnitude spectra of a thin shell, unimorph (-), in-phase bimorph ( $\cdots$ ), out-of-phase bimorph (- - ): (a) analytical predictions; (b) results from the experiment of Section 5

Figure 7: Schematic of the validation experiment for a shell with active patches and a cardboard liner, with measurement of acceleration and sound pressure responses given input from impulse hammer/shaker and/or active patches; refer to Appendix A for symbols

Figure 8: Measured transfer functions of a piezoelectric patch, indicating output strain per input voltage in both principal ( $\cdots$ ) and orthogonal (-) directions: (a) magnitude; (b) phase; (c) coherence

Figure 9: Effect of local control on the predicted transverse shell response under excitation of the $(2,3)$ mode: (a) uncontrolled; (b) controlled

Figure 10: Effect of global control on the predicted shell response under excitation of the $(2,3)$ mode: (a) varying magnitude from $0.1\left|\tilde{V}_{c}\right|$ to $10\left|\tilde{V}_{c}\right|$ with $\left|\tilde{V}_{c}\right|$ denoted by $\bullet$; (b) varying phase from $-180^{\circ}$ to $180^{\circ}$ with $\angle \tilde{V}_{c}$ denoted by $\bullet$; (c) controlled displacement profile 
Figure 11: Comparison of control transfer functions $\tilde{\Xi}(\omega)$ for a shell with patch at location $1(-)$ and $2(\cdots)$ : (a) global control scheme; (b) local control scheme

Figure 12: Schematics illustrating three patch locations and orientations, unrolled (top), and on a closed shell (bottom): (a) location 1; (b) location 2; (c) location 3

Figure 13: Effect of active patch location on the predicted global mean-square velocity of shell, $\Psi_{\dot{w}}^{2}$, as compared to uncontrolled $(-)$, where modal values are indicated by $(\bullet)$ : (a) location $1(\cdots)$; location $2(---)$; location $3(\bullet-\bullet-)$

Figure 14: Schematics illustrating three vibration control system designs: (a) design I, (b) design II, (c) design III

\section{List of Table Captions}

Table 1: Material properties and geometry of shell, liner, and active patches, along with force, motion, and control locations

Table 2: Predicted natural frequencies, modal loss factors, and insertion losses of unlined and lined shell structures for selected modes

Table 3: Summary of comparative vibration control studies from model: (a) in the context of global broadband performance; (b) for modal insertion losses (IL) of global velocity at selected modes

Table 4: Measured responses and insertion losses (IL) for local acceleration and sound pressure level (SPL) at a single mode 


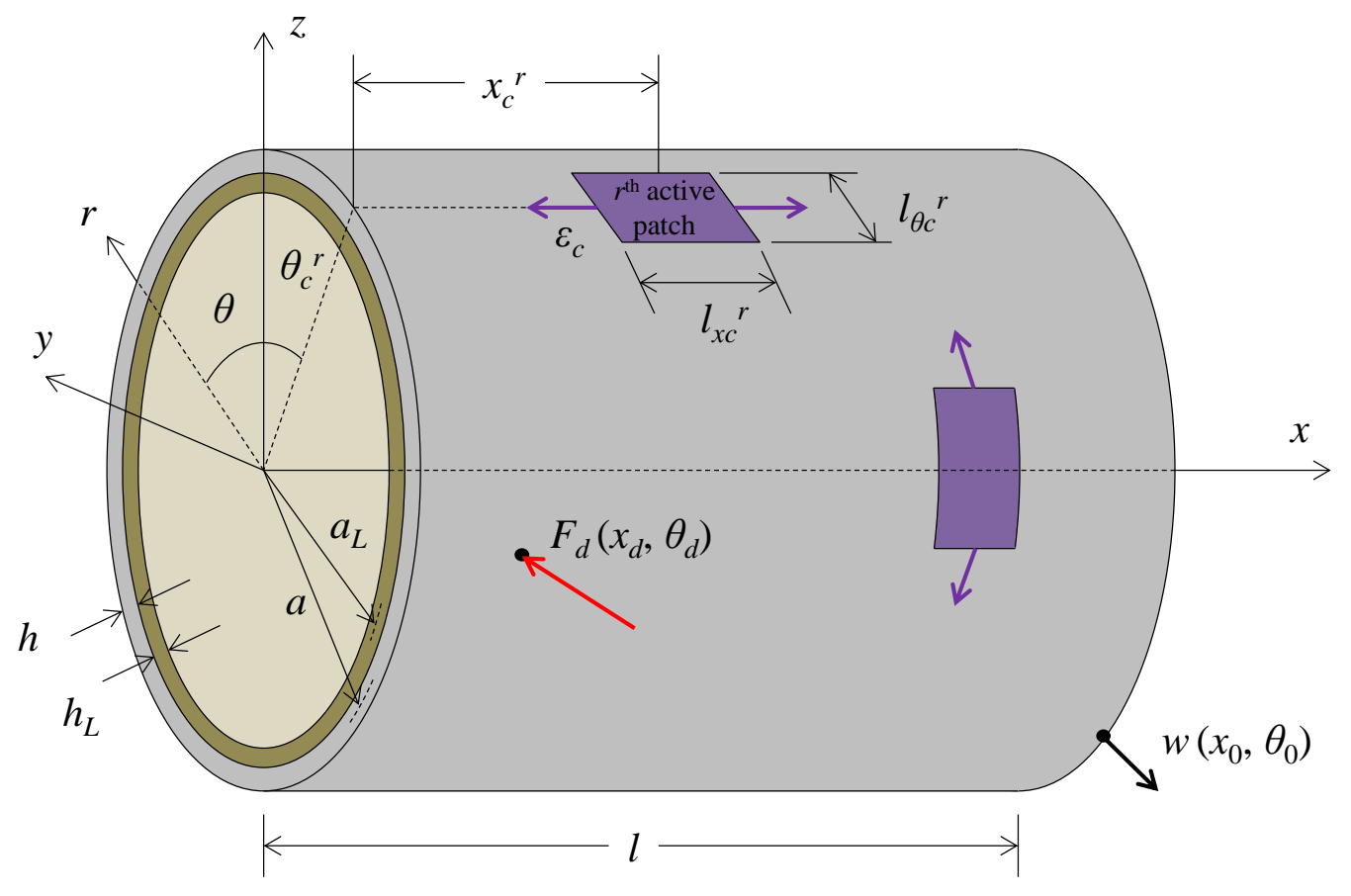

Figure 1: Example case: active patches and cardboard liner on a thin cylindrical shell with free boundaries (extension of the system studied in [1]); patch size/location and force/measurement location are labeled; see Appendix A and text for definition of symbols 
(a)

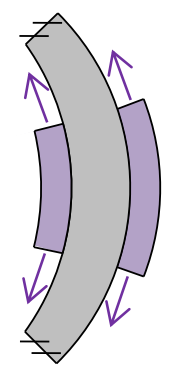

(b)

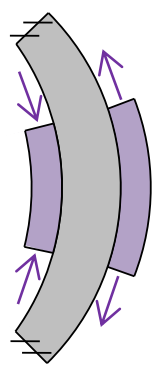

(c)

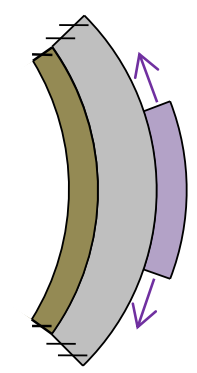

Figure 2: Schematics of shell sector (in the $\theta-r$ plane) with three configurations: (a) bimorph with in-phase actuation; (b) bimorph with out-of-phase actuation; (c) unimorph with cardboard liner 
(a)

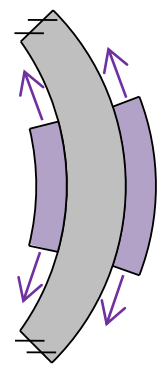

(b)

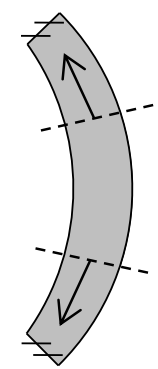

(c)

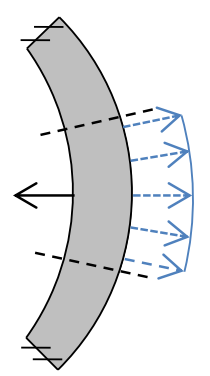

Figure 3: Effective distributed pressure loading: (a) in-phase actuation; (b) equivalent force loading; (c) resultant of equivalent force $(\leftarrow)$ needs to be balanced by an effective pressure $(---))$ 

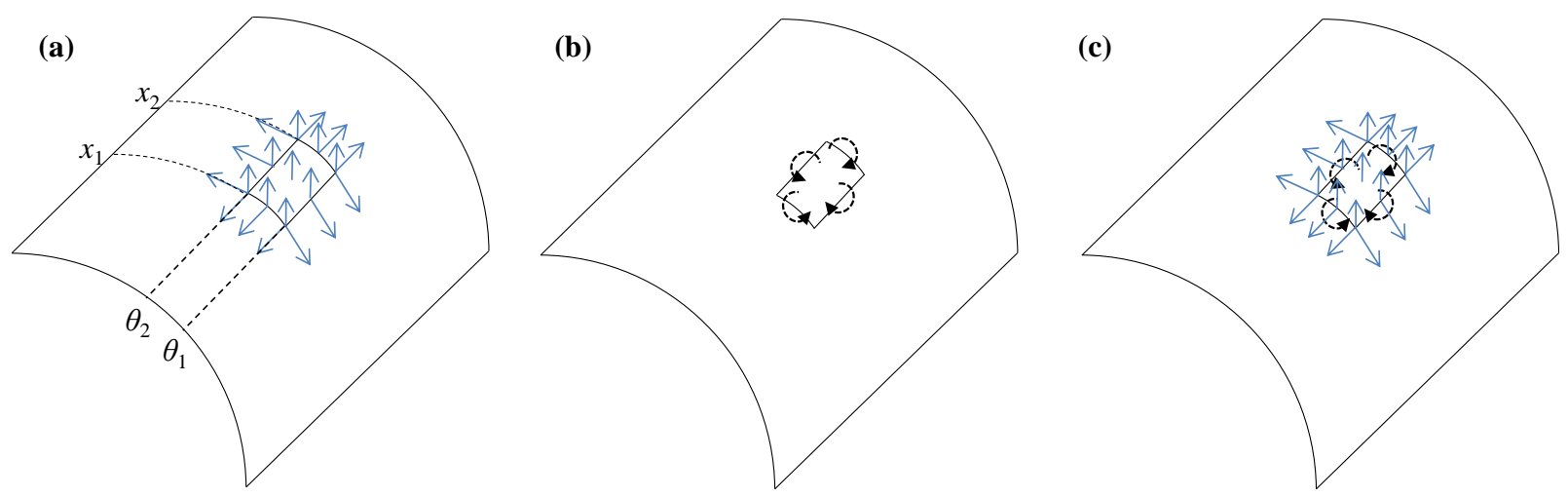

Figure 4: Equivalent loading from alternate active patch configurations on a shell section: (a) in-phase bimorph with $F_{x}, F_{\theta}$, and $P_{r}(\rightarrow)$ and patch endpoints labeled; (b) out-of-phase bimorph with $M_{x}$ and $M_{\theta}(---)$ ); (c) proposed combined loading representation of unimorph 

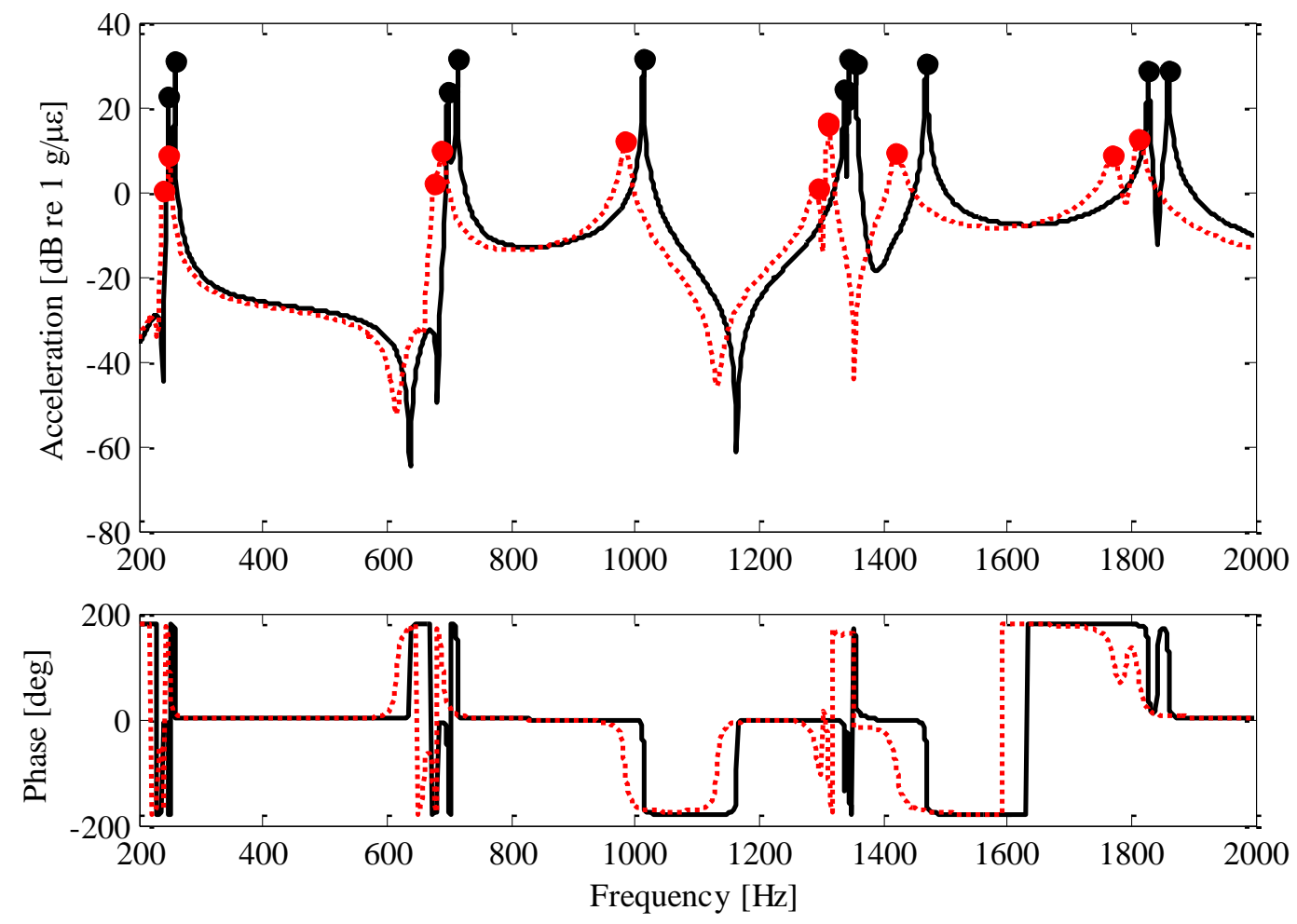

Figure 5: Effect of control input from an active patch on predicted vibratory response spectra of a thin shell $\left(\right.$ i.e. $\left.A_{c}(\omega)\right)$, unlined $(-)$, and lined $(\cdots)$, with modal values denoted by large dots $(\bullet)$ 

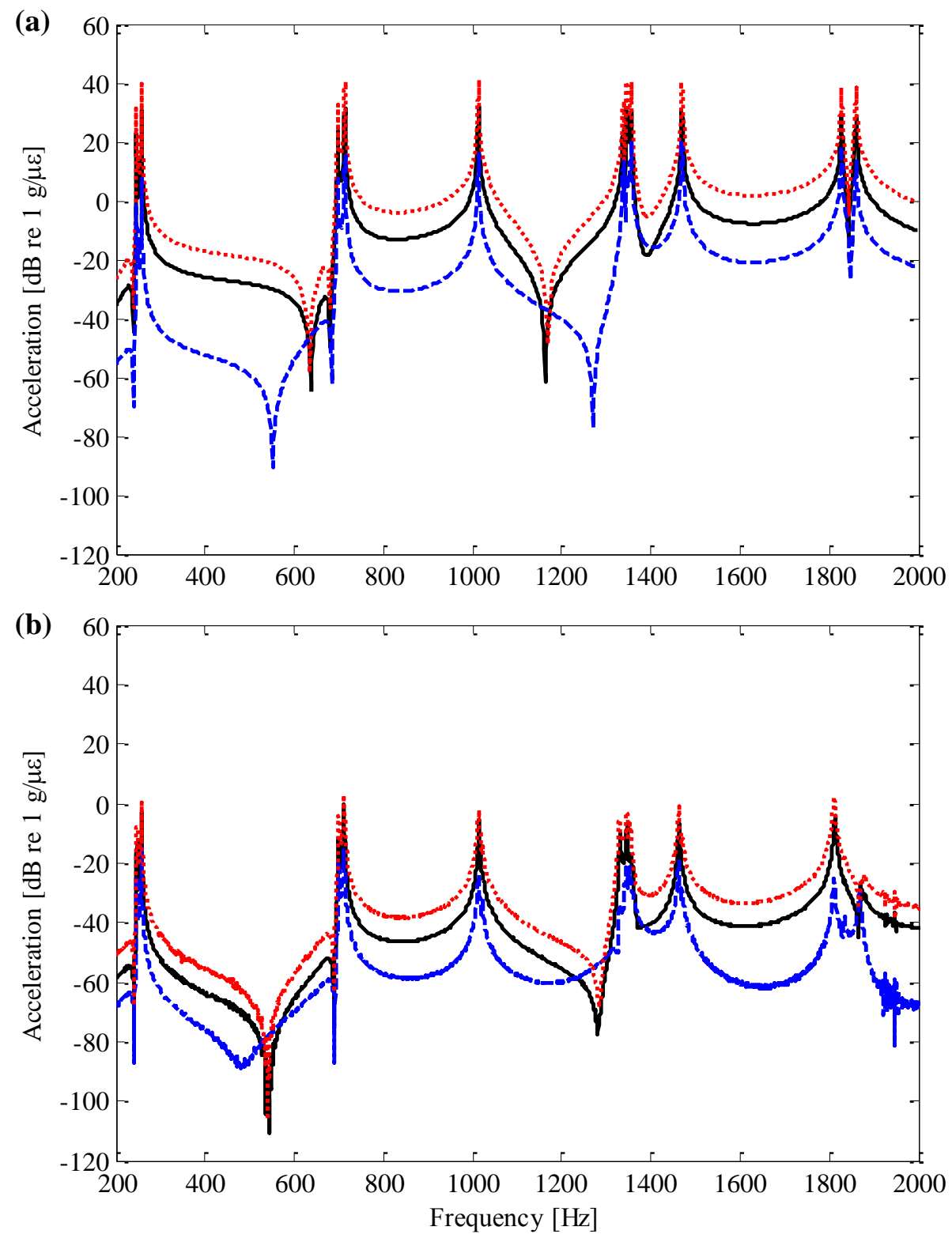

Figure 6: Comparison of unimorph and bimorph responses, in terms of $\tilde{A_{c}}(\omega)$ magnitude spectra of a thin shell, unimorph (-), in-phase bimorph ( $\cdots$ ), out-of-phase bimorph (- - ): (a) analytical predictions; (b) results from the experiment of Section 5 


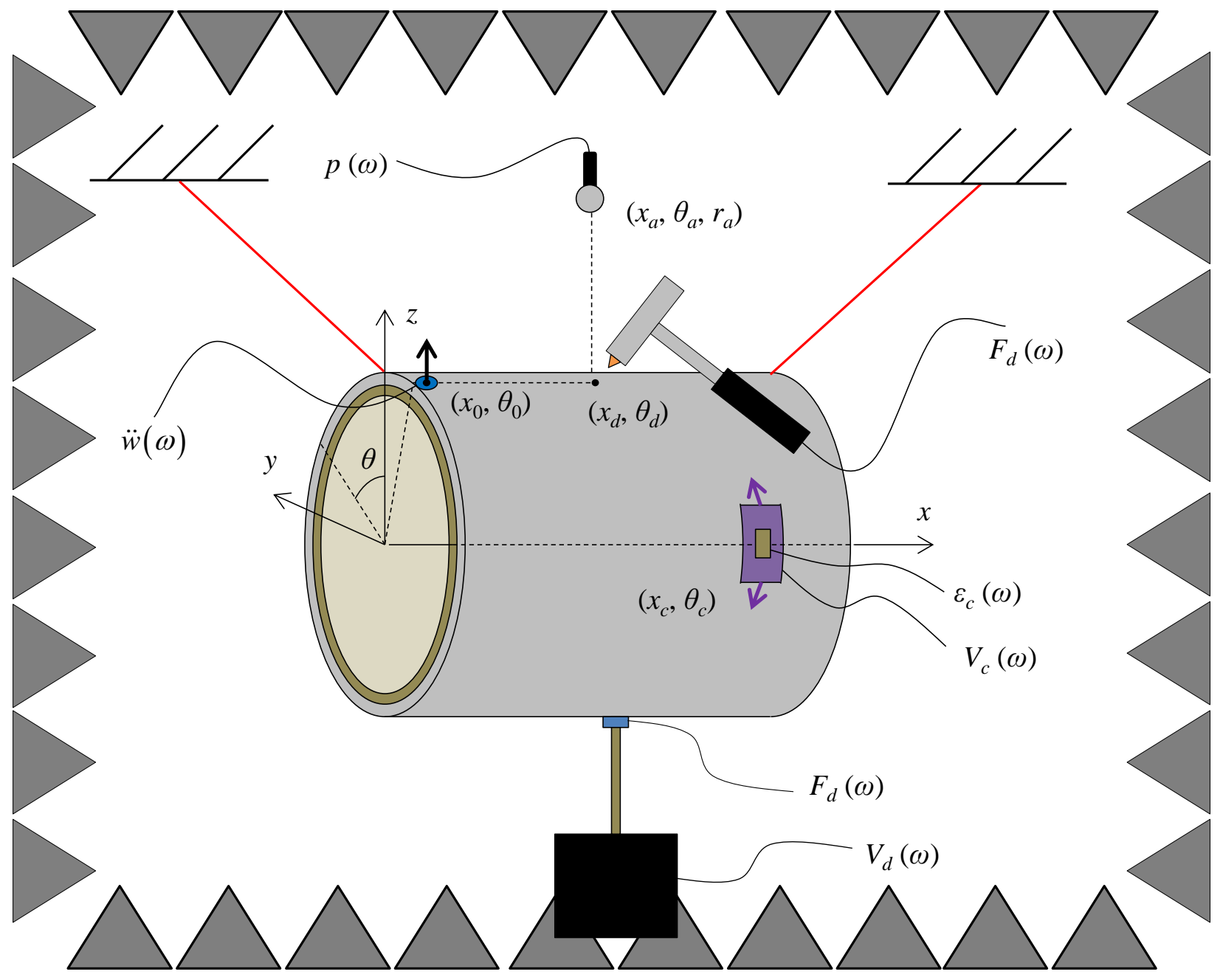

Figure 7: Schematic of the validation experiment for a shell with active patches and a cardboard liner, with measurement of acceleration and sound pressure responses given input from impulse hammer/shaker and/or active patches; refer to Appendix A for symbols 
(a)

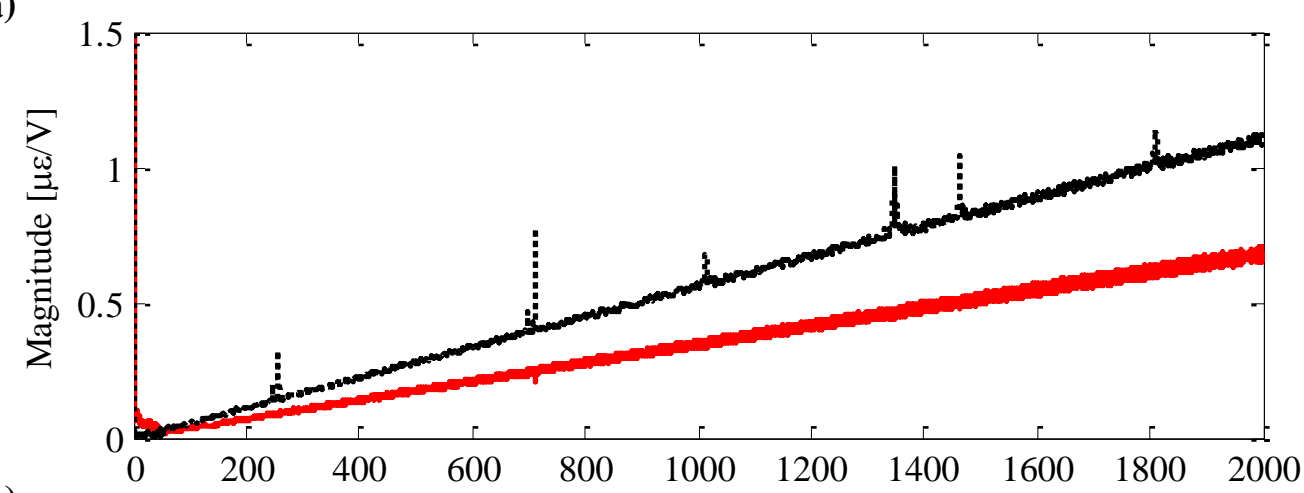

(b)

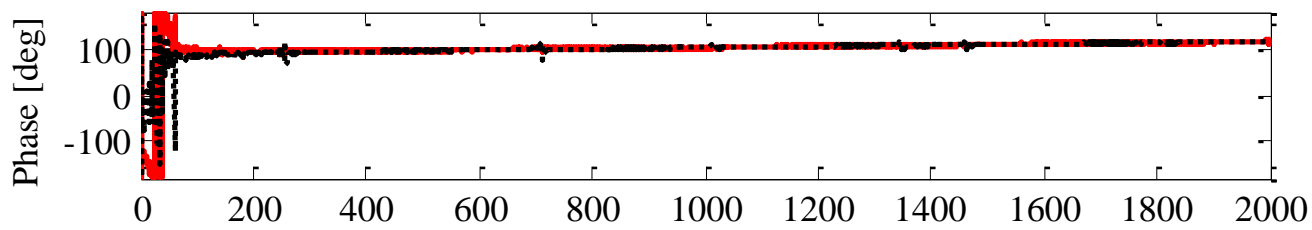

(c)

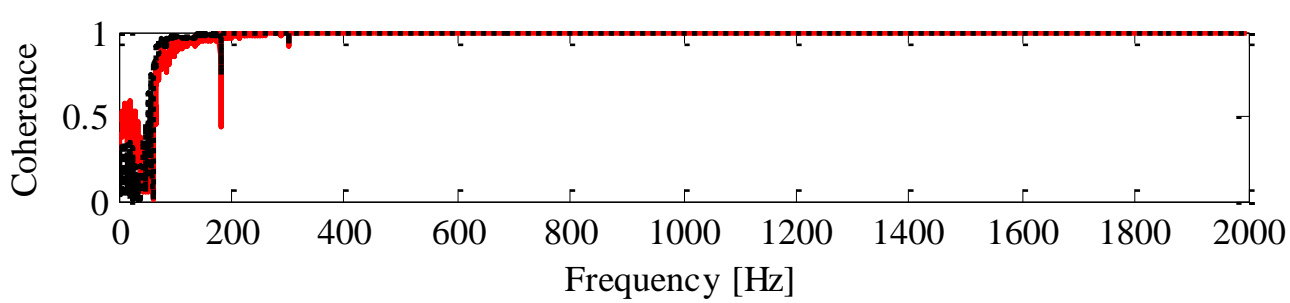

Figure 8: Measured transfer functions of a piezoelectric patch, indicating output strain per input voltage in both principal ( $\cdots$ ) and orthogonal (-) directions: (a) magnitude; (b) phase; (c) coherence 

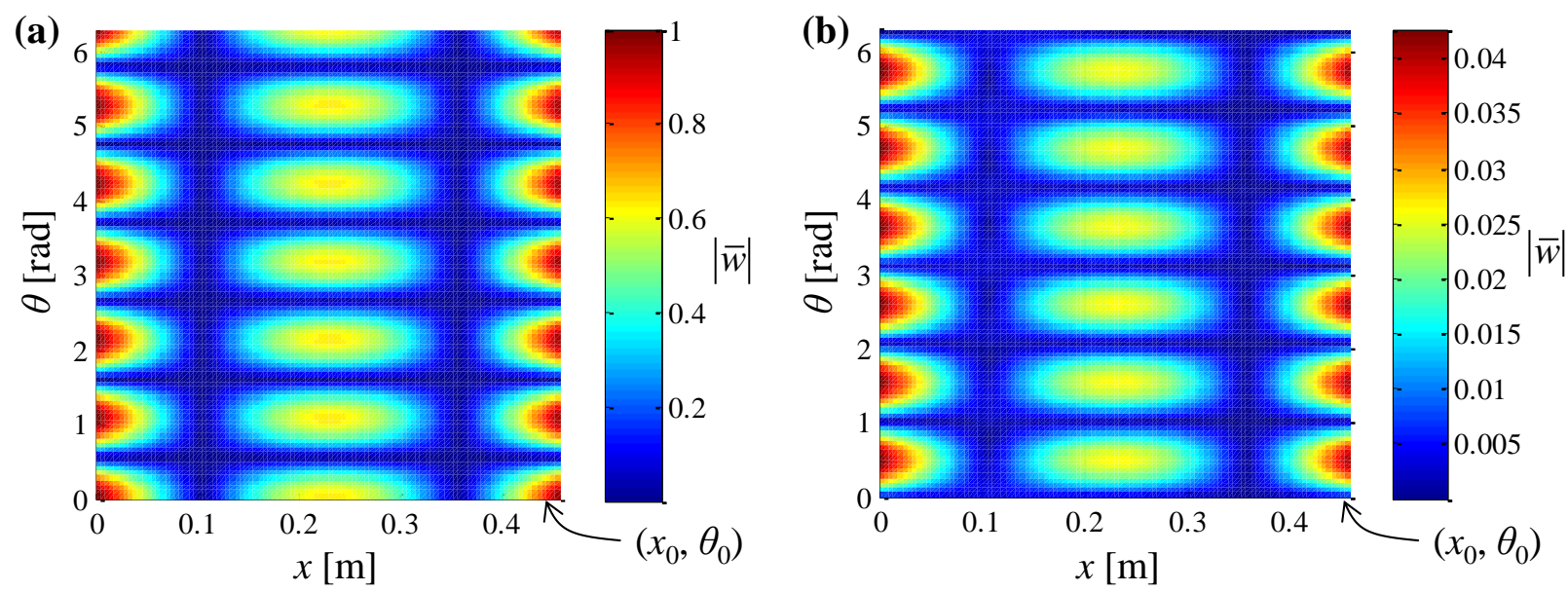

Figure 9: Effect of local control on the predicted transverse shell response under excitation of the $(2,3)$ mode: (a) uncontrolled; (b) controlled 
(a)
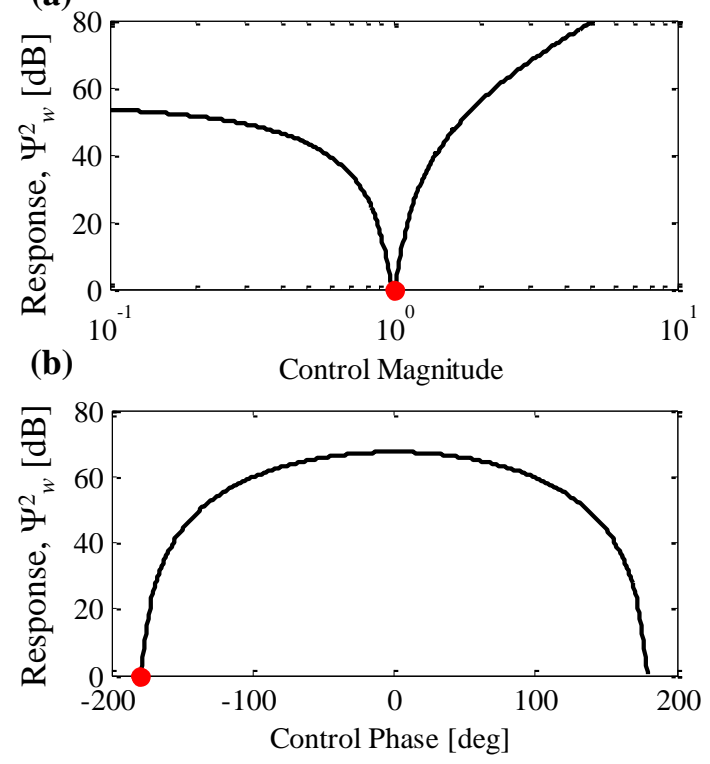

(c)

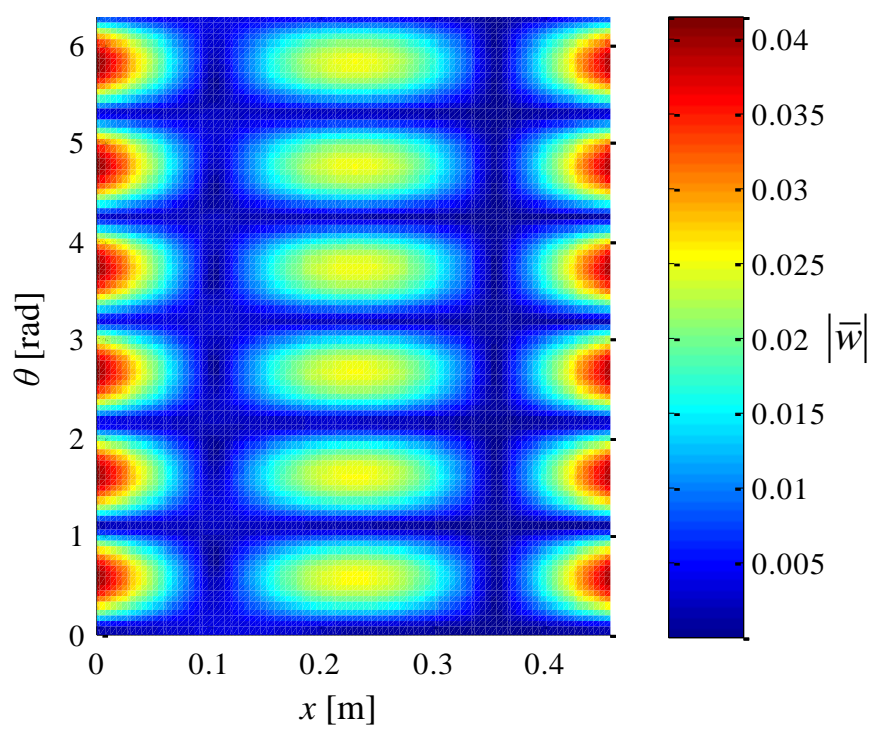

Figure 10: Effect of global control on the predicted shell response under excitation of the $(2,3)$ mode: (a) varying magnitude from $0.1\left|\tilde{V}_{c}\right|$ to $10\left|\tilde{V}_{c}\right|$ with $\left|\tilde{V}_{c}\right|$ denoted by $\bullet$; (b) varying phase from $-180^{\circ}$ to $180^{\circ}$ with $\angle \tilde{V}_{c}$ denoted by $\bullet$; (c) controlled displacement profile 

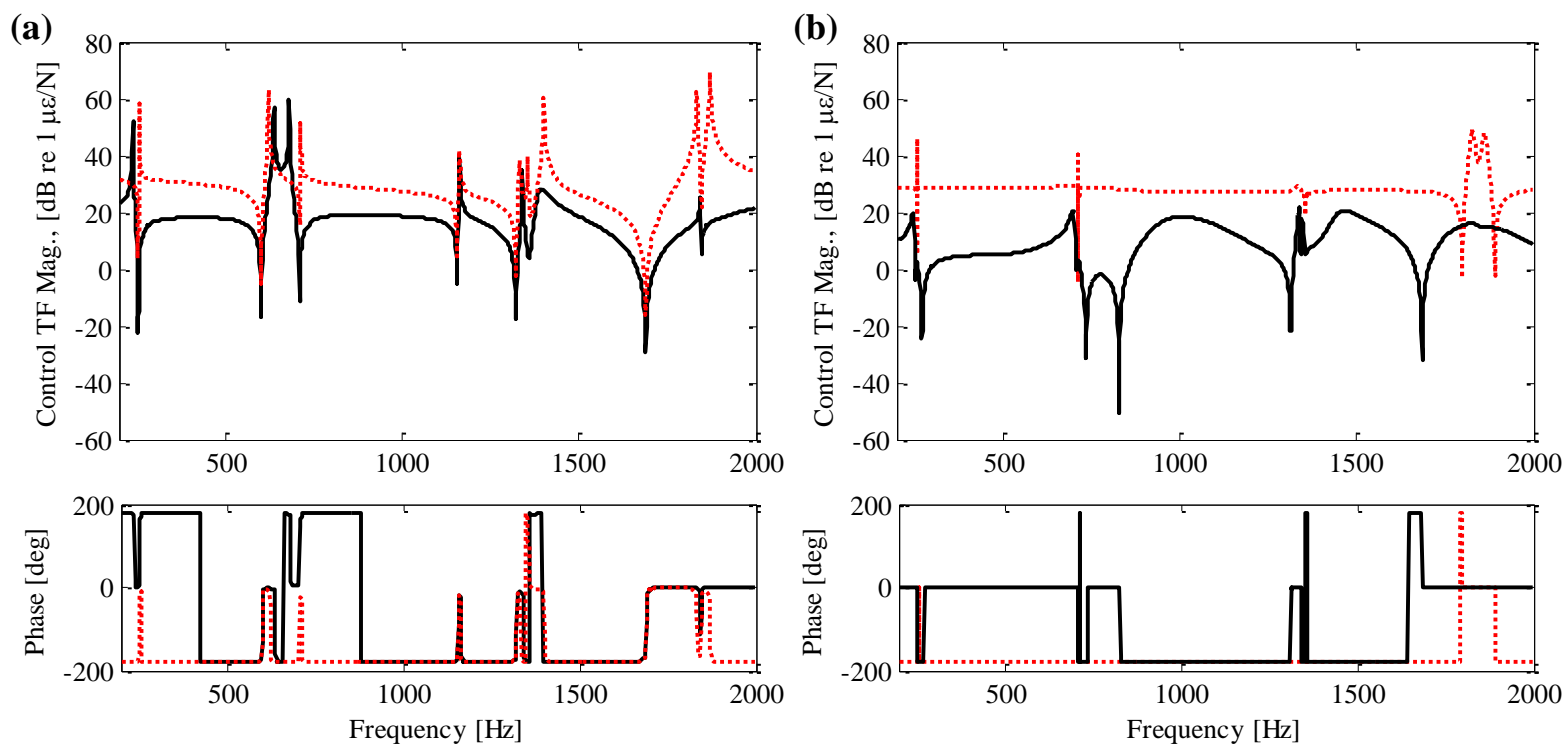

Figure 11: Comparison of control transfer functions $\tilde{\Xi}(\omega)$ for a shell with patch at location $1(-)$ and $2(\cdots)$ : (a) global control scheme; (b) local control scheme 
(a)
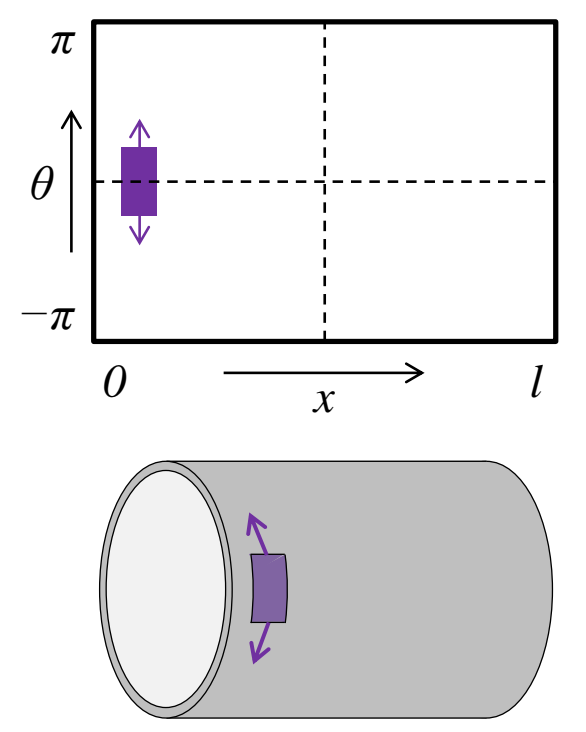

(b)
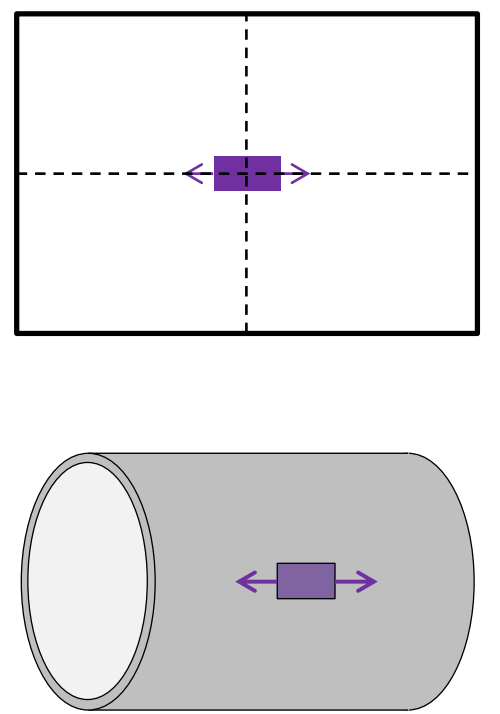

(c)
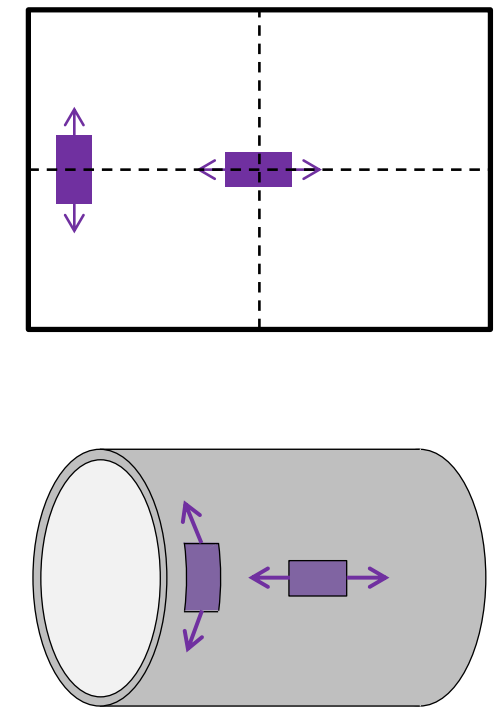

Figure 12: Schematics illustrating three patch locations and orientations, unrolled (top), and on a closed shell (bottom): (a) location 1; (b) location 2; (c) location 3 


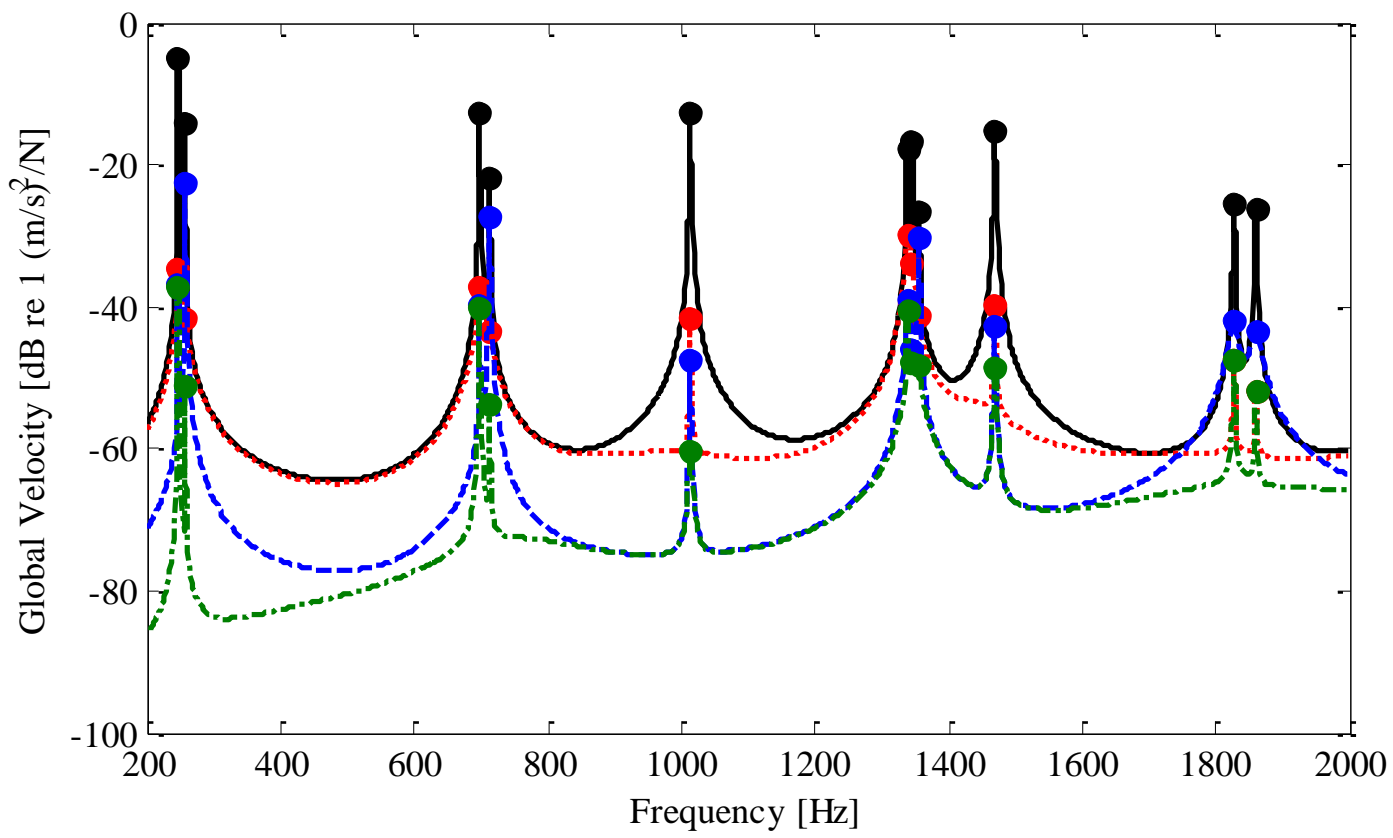

Figure 13: Effect of active patch location on the predicted global mean-square velocity of shell, $\Psi_{\dot{w}}^{2}$, as compared to uncontrolled $(-)$, where modal values are indicated by $(\bullet)$ : (a) location $1(\cdots)$; location $2(---)$; location $3(\bullet-\bullet)$ 
(a)

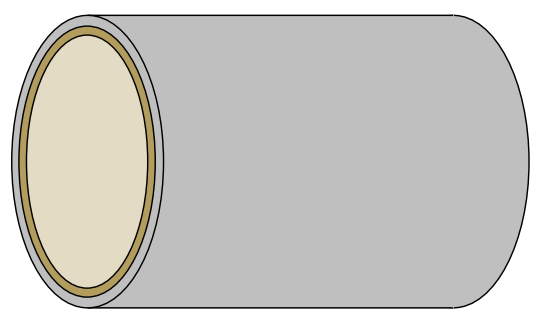

(b)

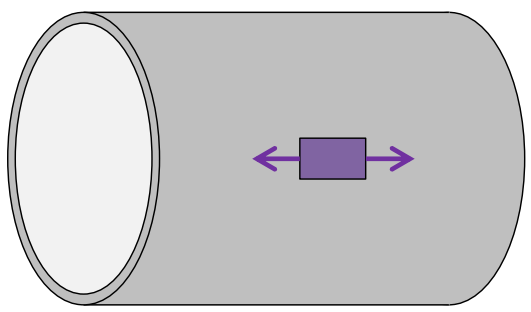

(c)

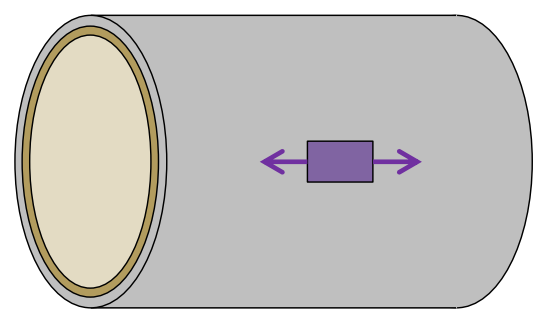

Figure 14: Schematics illustrating three vibration control system designs: (a) design I, (b) design II, (c) design III 
Table 1: Material properties and geometry of shell, liner, and active patches, along with force, motion, and control locations

\begin{tabular}{|c|c|c|c|c|}
\hline & & Shell & Passive Liner & Active Patch \\
\hline \multirow{5}{*}{$\begin{array}{l}\text { Material } \\
\text { Properties }\end{array}$} & Material & Aluminum & Cardboard & Composite \\
\hline & Young's Modulus, $E$ [GPa] & 68 & 4.9 & 31 \\
\hline & Density, $\rho\left[\mathrm{kg}-\mathrm{m}^{-3}\right]$ & 2606 & 649 & - \\
\hline & Poisson's Ratio, $v[-]$ & 0.33 & 0.30 & 0.35 \\
\hline & Material Loss Factor, $\eta[\%]$ & 0.10 & 3.40 & - \\
\hline \multirow{3}{*}{ Geometry } & Length, $l[\mathrm{~mm}]$ & 458 & 458 & $28.3 \times 14.8$ \\
\hline & Thickness, $h[\mathrm{~mm}]$ & 2.10 & 0.62 & 0.30 \\
\hline & Radius, $a[\mathrm{~mm}]$ & 75.4 & 74.0 & 76.6 \\
\hline \multirow{3}{*}{ Locations } & Force (Excitation) Location, $\left(\bar{x}_{d}, \bar{\theta}_{d}\right)$ & \multicolumn{3}{|c|}{$(0.56,0.0)$} \\
\hline & Motion (Response) Location, $\left(\bar{x}_{0}, \bar{\theta}_{0}\right)$ & \multicolumn{3}{|c|}{$(0.98,0.0)$} \\
\hline & $\begin{array}{l}\text { Active Patch (Control) Locations, } \\
\qquad\left(\bar{x}_{c}, \bar{\theta}_{c}\right)\end{array}$ & \multicolumn{3}{|c|}{$(0.5,0.0) ;(0.05,0)$} \\
\hline
\end{tabular}


Table 2: Predicted natural frequencies, modal loss factors, and insertion losses of unlined and lined shell structures for selected modes

\begin{tabular}{ccccccc}
\hline \multirow{2}{*}{$\begin{array}{c}\text { Mode } \\
\#\end{array}$} & $\begin{array}{c}\text { Modal Index } \\
(m, n)\end{array}$ & \multicolumn{2}{c}{$\begin{array}{c}\text { Natural Frequency } \\
{[\mathrm{Hz}]}\end{array}$} & $\begin{array}{c}\text { Modal Loss Factor } \\
{[\%]}\end{array}$ & $\begin{array}{c}\text { Peak Insertion } \\
\text { Loss [dB] } \\
\text { [Lined) }\end{array}$ \\
\cline { 3 - 5 } & & Unlined & Lined & Unlined & Lined & \\
\hline 3 & $(0,3)$ & 698 & 675 & 0.1 & 1.1 & 22 \\
4 & $(1,3)$ & 712 & 690 & 0.1 & 1.1 & 18 \\
5 & $(2,3)$ & 1013 & 984 & 0.1 & 0.7 & 20 \\
6 & $(0,4)$ & 1338 & 1294 & 0.1 & 0.9 & 21 \\
9 & $(2,4)$ & 1469 & 1422 & 0.1 & 0.9 & 21 \\
\hline
\end{tabular}


Table 3: Summary of comparative vibration control studies from model: (a) in the context of global broadband performance; (b) for modal insertion losses (IL) of global velocity at selected modes

(a)

\begin{tabular}{|c|c|c|c|c|c|c|}
\hline \multirow[b]{2}{*}{$\begin{array}{l}\text { Design } \\
\text { Case }\end{array}$} & \multirow{2}{*}{$\begin{array}{l}\text { Active Patch } \\
\text { Location } \\
\text { (Fig. 11) }\end{array}$} & \multirow[b]{2}{*}{$\begin{array}{c}\text { Passive } \\
\text { Liner }\end{array}$} & \multicolumn{2}{|c|}{ Attenuation Metric $\Pi_{1}[\%]$} & \multicolumn{2}{|c|}{ Efficiency Metric $\Pi_{2}[-]$} \\
\hline & & & $\begin{array}{c}\text { Relative to } \\
\text { Unlined Shell }\end{array}$ & $\begin{array}{l}\text { Relative to } \\
\text { Lined Shell }\end{array}$ & $\begin{array}{c}\text { Relative to } \\
\text { Unlined Shell }\end{array}$ & $\begin{array}{l}\text { Relative to } \\
\text { Lined Shell }\end{array}$ \\
\hline I & NA & Yes & 89.6 & NA & NA & NA \\
\hline II & 2 & No & 97.0 & 68.7 & 0.470 & 0.058 \\
\hline III & 2 & Yes & 98.9 & 90.2 & 0.637 & 0.143 \\
\hline
\end{tabular}

(b)

\begin{tabular}{ccccc} 
Mode & Modal Index & \multicolumn{3}{c}{ Insertion Loss (IL) [dB] } \\
\cline { 3 - 5 }$\#$ & $(m, n)$ & Case I & Case II & Case III \\
\hline 3 & $(0,3)$ & 22 & 28 & 45 \\
4 & $(1,3)$ & 18 & 1 & 21 \\
5 & $(2,3)$ & 20 & 34 & 53 \\
6 & $(0,4)$ & 21 & 22 & 34 \\
9 & $(2,4)$ & 21 & 27 & 46 \\
\hline
\end{tabular}


Table 4: Measured responses and insertion losses (IL) for local acceleration and sound pressure level (SPL) at a single mode

\begin{tabular}{|c|c|c|c|c|c|c|c|}
\hline \multicolumn{2}{|r|}{ Cases } & $\begin{array}{c}f \\
{[\mathrm{~Hz}]}\end{array}$ & $\begin{array}{l}\text { Voltage Input } \\
\text { [V (deg.)] }\end{array}$ & $\begin{array}{c}\ddot{w}_{0} \\
{[\mathrm{~dB} \text { re } 1 \mathrm{~g}]}\end{array}$ & $\begin{array}{c}p_{a} \\
{[\mathrm{~dB} \text { re } 20 \mu \mathrm{Pa}]}\end{array}$ & $\begin{array}{l}\text { Accel. } \\
\text { IL }[\mathrm{dB}]\end{array}$ & $\begin{array}{c}\text { SPL } \\
\text { IL }[\mathrm{dB}]\end{array}$ \\
\hline \multirow{3}{*}{$\begin{array}{c}\text { Unlined } \\
\text { Shell }\end{array}$} & $\begin{array}{c}\text { Disturbance } \\
\left(F_{d}=0.016 \mathrm{~N}\right)\end{array}$ & 993 & $0.18\left(0^{\circ}\right)$ & 14 & 70 & NA & NA \\
\hline & $\begin{array}{l}\text { Controlled } \\
\text { (for Accel.) }\end{array}$ & 993 & $4.10\left(163^{\circ}\right)$ & -37 & 54 & $51^{\dagger}$ & 16 \\
\hline & $\begin{array}{l}\text { Controlled } \\
\text { (for SPL) }\end{array}$ & 993 & $4.00\left(170^{\circ}\right)$ & -3 & 28 & 17 & $42^{\dagger}$ \\
\hline \multirow{3}{*}{$\begin{array}{l}\text { Lined } \\
\text { Shell }\end{array}$} & $\begin{array}{c}\text { Disturbance } \\
\left(F_{d}=0.016 \mathrm{~N}\right)\end{array}$ & 968 & $0.22\left(0^{\circ}\right)$ & 8 & 62 & $6^{*}$ & $8^{*}$ \\
\hline & $\begin{array}{l}\text { Controlled } \\
\text { (for Accel.) }\end{array}$ & 968 & $4.40\left(153.5^{\circ}\right)$ & -34 & 52 & $48^{\ddagger}$ & 18 \\
\hline & $\begin{array}{l}\text { Controlled } \\
\text { (for SPL) }\end{array}$ & 968 & $4.23\left(171^{\circ}\right)$ & -3 & 27 & 17 & $43^{\ddagger}$ \\
\hline
\end{tabular}

* passive: design I

$\dagger$ active: design II

+ concurrent: design III 